\title{
Research Article \\ Optimal Portfolios in Lévy Markets under State-Dependent Bounded Utility Functions
}

\author{
José E. Figueroa-López ${ }^{1}$ and Jin $\mathbf{M a}^{2}$ \\ ${ }^{1}$ Department of Statistics, Purdue University, West Lafayette, IN 47906, USA \\ ${ }^{2}$ Department of Mathematics, University of Southern California, Los Angeles, CA 90089, USA
}

Correspondence should be addressed to José E. Figueroa-López, figueroa@stat.purdue.edu

Received 18 August 2009; Accepted 28 January 2010

Academic Editor: Vo V. Anh

Copyright (C 2010 J. E. Figueroa-López and J. Ma. This is an open access article distributed under the Creative Commons Attribution License, which permits unrestricted use, distribution, and reproduction in any medium, provided the original work is properly cited.

\begin{abstract}
Motivated by the so-called shortfall risk minimization problem, we consider Merton's portfolio optimization problem in a non-Markovian market driven by a Lévy process, with a bounded state-dependent utility function. Following the usual dual variational approach, we show that the domain of the dual problem enjoys an explicit "parametrization," built on a multiplicative optional decomposition for nonnegative supermartingales due to Föllmer and Kramkov (1997). As a key step we prove a closure property for integrals with respect to a fixed Poisson random measure, extending a result by Mémin (1980). In the case where either the Lévy measure $v$ of $Z$ has finite number of atoms or $\Delta S_{t} / S_{t^{-}}=\zeta_{t} \vartheta\left(\Delta Z_{t}\right)$ for a process $\zeta$ and a deterministic function $\vartheta$, we characterize explicitly the admissible trading strategies and show that the dual solution is a risk-neutral local martingale.
\end{abstract}

\section{Introduction}

The task of determining good trading strategies is a fundamental problem in mathematical finance. A typical approach to this problem aims at finding the trading strategy that maximizes, for example, the final expected utility, which is defined as a concave and increasing function $U: \mathbb{R} \rightarrow \mathbb{R} \cup\{-\infty\}$ of the final wealth. There are, however, many applications where a utility function varies with the underlying securities, or even random. For example, if the market is incomplete, it is often more beneficial to allow certain degree of "shortfall" in order to reduce the "super-hedging cost" (see, e.g., [1, 2] for more details). Mathematically, such a shortfall risk is often quantified by the expected loss

$$
\mathbb{E}\left[L\left(\left(H-V_{T}\right)^{+}\right)\right],
$$

where $L: \mathbb{R}_{+} \rightarrow \mathbb{R}$ is a convex increasing "loss" function, $H$ is a contingent claim, and $V$ is the value process that is subject to the constraint $V_{0} \leq z$, for a given initial endowment $z \geq 0$. 
The above shortfall minimizing problem can be easily recast as a utility maximization problem with a bounded state-dependent utility of the form

$$
U(v ; \omega):=L(H(\omega))-L\left((H(\omega)-v)^{+}\right), \quad \omega \in \Omega,
$$

as it was first pointed out by Föllmer and Leukert [3] (see Definition 2.3 for a formal description of the general bounded state-dependent utility). Then, the minimal shortfall risk cost is given by

$$
u(z):=\sup \left\{\mathbb{E}\left[U\left(V_{T}(\cdot), \cdot\right)\right]: V \text { is admissible and } V_{0} \leq z\right\}, \quad z \geq 0,
$$

where the supremum is taken over all wealth processes $\left\{V_{t}\right\}_{t \leq T}$ generated by admissible trading strategies. We should point out here that it is the boundedness and potential nondifferentiability of such utility function that give rise to some technical issues which make the problem interesting.

The existence and essential uniqueness of the solution to the problem (1.3) in its various special forms have been studied by many authors see, for example, Cvitanić [4], Föllmer and Leukert [3], Xu [5], and Karatzas and Žitković [6], to mention a few. However, while the convex duality approach in [3] succeeds in dealing with the non-Markovian nature of the model, it does not seem to shed any light on how to compute, in a feasible manner, the optimal trading strategy, partly due to the generality of the model considered there. In this paper we will consider a specific but popular model driven by a Lévy process. Our goal is to narrow down the domain of dual problem so that the convex duality method holds true. Furthermore, we will try to give an explicit construction of the dual domain that contains the dual optimizer. Although at this point our results are still rather general, and at a theoretical level, we believe that this is a necessary step towards a feasible computational implementation of the convex duality method.

While the utility maximization problem of this kind can be traced back to Merton $[7,8]$, in this paper we shall follow the convex duality method, suggested by Karatzas et al. [9], and later extended by Kunita [10] to general Lévy market models. However, we note that in [9] the utility function was required to be unbounded, strictly increasing and concave, continuously differentiable, and other technical assumptions including the so-called Inada conditions. On the other hand, since one of the key tools in [10] is an exponential representation for positive local supermartingales (see, e.g., [11, Lemma 4.2]), it is required that the utility function satisfies the same conditions as in [9] (in particular, unboundedness), plus that the dual domain $\Gamma$ contains all positive "risk-neutral" local supermartingales. The boundedness and potential nondifferentiability of the utility function in our case thus cause some technical subtleties. For example, the dual optimal process can be 0 with positive probability, thus the representation theorem of Kunita [11, Lemma4.2] does not apply anymore.

A key element that we use to overcome these technical difficulties is an exponential representation theorem for nonnegative supermartingales by Föllmer and Kramkov [12]. This result leads to an explicit construction of the dual domain, based on those nonnegative supermartingales that can be written as stochastic exponentials $\xi=\xi_{0} \varepsilon(X-A)$, with $A$ being an increasing process and $X$ belonging to a class of semimartingales $\mathcal{S}$ that is closed under Émery's topology. To validate this approach we prove a closure property for integrals with respect to a fixed compensated Poisson random measures, a result of interest on its 
own, which extends the analog property for integrals with respect to a fixed semimartingale due to Mémin [13]. Finally, unlike some previous works on the subject (see, e.g., Föllmer and Leukert [3] and $\mathrm{Xu}$ [5]), we do not use the so-called bipolar theorem of Kramkov and Schachermayer [14] to argue the attainability of the optimal final wealth. Instead, we shall rely on the fundamental characterization of contingent claims that are super replicable [1,2], reducing the problem of finding the optimal primal solution to a super-eplication problem.

We believe that the dual problem proposed in this paper offers several advantages. For example, since the dual class enjoys a fairly "explicit" description and "parametrization," our results could be considered as a first step towards a feasible computational implementation of the covex duality method. Furthermore, the specific results we obtained for the Lévy market can be used to characterize the elements of the dual domain and the admissible trading strategies. In particular, if either (i) the jumps of the price process $S$ are driven by the superposition of finitely many shot-noise Poisson processes, or (ii) $\Delta S_{t} / S_{t^{-}}=\zeta_{t} \vartheta\left(\Delta Z_{t}\right)$ for a process $\zeta$ and a deterministic function $\vartheta$, we show that the dual solution is a risk-neutral local martingale.

We would like to remark that some of our results are related to those in $\mathrm{Xu}$ [5], but there are essential differences. For example, the model in [5] exhibits only finite-jump activity and allows only downward price jumps (in fact, this assumption seems to be important for the approach there), while our model allows for general jump dynamics, and our approach is also valid for general additive processes, including the time-inhomogeneous cases considered in [5] (see (ii) of Section 6).

The rest of the paper is organized as follows. In Section 2 we introduce the financial model, along with some basic terminology that will be used throughout the paper. The convex duality method is revised in Section 3, where a potential optimal final wealth is constructed. An explicit description of a dual class and a characterizations of the dual optimum and admissible trading strategies are presented in Section 4. In Section 5 we show that the potential optimal final wealth is attained by an admissible trading strategy, completing the proof of the existence of optimal portfolio. In Section 6 we give some concluding remarks. Some necessary fundamental theoretical results, such as the exponential representation for nonnegative supermartingales of Föllmer and Kramkov [12] and the closure property for integrals with respect to Poisson random measures, are collected in Appendix A. Finally, Appendix B briefly outlines the proofs of the convex duality results used in the paper.

\section{Notation and Problem Formulation}

Throughout this paper we assume that all the randomness comes from a complete probability space $(\Omega, \mathcal{F}, \mathbb{P})$, on which there is defined a Lévy process $Z$ with Lévy triplet $\left(\sigma^{2}, v, 0\right)$ (see Sato [15] for the terminology). By the Lévy-Itô decomposition, there exist a standard Brownian motion $W$ and an independent Poisson random measure $N$ on $\mathbb{R}_{+} \times \mathbb{R} \backslash\{0\}$ with mean measure $\mathbb{E} N(d t, d z)=v(d z) d t$, such that

$$
Z_{t}=\sigma W_{t}+\int_{0}^{t} \int_{|z| \leq 1} z \widetilde{N}(d s, d z)+\int_{0}^{t} \int_{|z|>1} z N(d s, d z),
$$

where $\widetilde{N}(d t, d z):=N(d t, d z)-v(d z) d t$. Let $\mathbb{F}:=\left\{\mathcal{F}_{t}\right\}_{t \geq 0}$ be the natural filtration generated by $W$ and $N$, augmented by all the null sets in $\mathcal{F}$ so that it satisfies the usual conditions (see, e.g., [16]). 


\subsection{The Market Model}

We assume that there are two assets in the market: a risk free bond (or money market account), and a risky asset, say, a stock. The case of multiple stocks, such as the one studied in [10], can be treated in a similar way without substantial difficulties (see Section 6 for more details). As it is customary all the processes are taken to be discounted to the present value so that the value $B_{t}$ of the risk-free asset can be assumed to be identically equal to 1 . The (discounted) price of the stock follows the stochastic differential equation

$$
d S_{t}=S_{t^{-}}\left\{b_{t} d t+\sigma_{t} d W_{t}+\int_{\mathbb{R}_{0}} v(t, z) \widetilde{N}(d t, d z)\right\}
$$

where $\mathbb{R}_{0}:=\mathbb{R} \backslash\{0\}, b \in L_{\text {loc' }}^{1} \sigma \in L_{\text {loc }}^{2}(W)$, and $v \in G_{\text {loc }}(N)$ (see [17] for the terminology). More precisely, $b, \sigma$, and $v$ are predictable processes such that $v(\cdot, \cdot)>-1$ a.s. (hence, $S$. $>0$ a.s.), and that the processes

$$
\left\{\int_{0}^{t}\left|b_{s}\right| d s\right\}_{t \geq 0}, \quad\left\{\int_{0}^{t}\left|\sigma_{s}\right|^{2} d s\right\}_{t \geq 0}, \quad\left\{\left(\sum_{u \leq t} v^{2}\left(u, \Delta Z_{u}\right)\right)^{1 / 2}\right\}_{t \geq 0}
$$

are locally integrable with respect to time. Even though we will work with a finite horizon $[0, T]$ later on, we choose to define our market model on $\mathbb{R}_{+}$. Finally, we assume that the market is free of arbitrage so that there exists at least one risk-neutral probability measure $\mathbb{Q}$ such that the (discounted) process $S_{t}, 0 \leq t \leq T$, is an $\mathbb{F}$-local martingale under $\mathbb{Q}$. Throughout, $\mathcal{M}$ will stand for the class of all equivalent risk neutral measures $\mathbb{Q}$. It is relevant to mention that we do not impose market completeness, and hence, the class $\mathcal{M}$ is not assumed to be a singleton.

\subsection{Admissible Trading Strategies and the Utility Maximization Problem}

A trading strategy is determined by a predictable locally bounded process $\beta$ representing the proportion of total wealth invested in the stock. Then, the resulting wealth process is governed by the stochastic differential equation

$$
V_{t}=w+\int_{0}^{t} V_{s^{-}} \frac{\beta_{s}}{S_{s^{-}}} d S_{s}, \quad 0<t \leq T,
$$

where $w$ stands for the initial endowment. For future reference, we give a precise definition of "admissible strategies."

Definition 2.1. The process $V^{w, \beta}:=V$ solving (2.4) is called the value process corresponding to the self-financing portfolio with initial endowment $w$ and trading strategy $\beta$. We say that a value process $V^{w, \beta}$ is "admissible" or that the process $\beta$ is "admissible" for $w$ if $V_{t}^{w, \beta} \geq$ 0 , for all $t \in[0, T]$.

For a given initial endowment $w$, we denote the set all admissible strategies for $w$ by $\mathcal{U}_{\mathrm{ad}}^{w}$, and the set of all admissible value processes by $\mathcal{V}_{\mathrm{ad}}^{w}$. In light of the Doléans-Dade 
stochastic exponential of semimartingales (see, e.g., [17, Section I.4f]), one can easily obtain necessary and sufficient conditions for admissibility.

Proposition 2.2. A predictable locally bounded process $\beta$ is admissible if and only if

$$
\mathbb{P}\left[\left\{\omega \in \Omega: \beta_{t} v\left(t, \Delta Z_{t}\right) \geq-1, \text { for a.e. } t \leq T\right\}\right]=1 .
$$

To define our utility maximization problem, we begin by introducing the bounded statedependent utility function.

Definition 2.3. A random function $U: \mathbb{R}_{+} \times \Omega \mapsto \mathbb{R}_{+}$is called a "bounded and state-dependent utility function" if

(1) $U(\cdot, \omega)$ is nonnegative, nondecreasing, and continuous on $[0, \infty)$;

(2) for each fixed $w$, the mapping $\omega \mapsto U(w, \omega)$ is $\mathcal{F}_{T}$-measurable;

(3) there is an $\mathcal{F}_{T}$-measurable, positive random variable $H$ such that for all $\omega \in \Omega$, $U(\cdot, \omega)$ is a strictly concave differentiable function on $(0, H(\omega))$, and it holds that

$$
\begin{aligned}
U(w, \omega) \equiv & U(w \wedge H(\omega), \omega), \quad w \in \mathbb{R}_{+}, \\
& \mathbb{E}[U(H ; \cdot)]<\infty .
\end{aligned}
$$

Notice that the $\mathscr{F}_{T}$-measurability of the random variable $\omega \rightarrow U\left(V_{T}(\omega), \omega\right)$ is automatic because $U(w, \omega)$ is $B([0, \infty)) \times \mathcal{F}_{T}$-measurable in light of the above conditions (1) and (2). We remark that while assumption (2.7) is merely technical, assumption (2.6) is motivated by the shortfall risk measure (1.2). Our utility optimization problem is thus defined as

$$
u(z):=\sup \left\{\mathbb{E}\left[U\left(V_{T}(\cdot), \cdot\right)\right]: V \in \mathcal{V}_{\text {ad }}^{w} \text { with } w \leq z\right\}
$$

for any $z>0$. We should note that the above problem is relevant only for those initial wealths $z$ that are smaller than $\bar{w}:=\sup _{\mathbb{Q} \in \mathcal{M}} \mathbb{E}_{\mathbb{Q}}\{H\}$, the super-hedging cost of $H$. Indeed, if $z \geq \bar{w}$, then there exists an admissible trading strategy $\beta^{*}$ for $z$ such that $V_{T}^{z, \beta^{*}} \geq H$ almost surely, and consequently, $u(z)=\mathbb{E}[U(H, \cdot)]$ (see $[1,2]$ for this super-hedging result).

Our main objectives in the rest of the paper are the following: (1) Define the dual problem and identify the relation between the value functions of the primal and the dual problems; (2) By suitably defining the dual domain, prove the attainability of the associated dual problem; (3) Show that the potential optimum final wealth induced by the minimizer of the dual problem can be realized by an admissible portfolio. We shall carry out these tasks in the remaining sections.

\section{The Convex Duality Method and the Dual Problems}

In this section we introduce the dual problems corresponding to the primal problem (1.3) and revise some standard results of convex duality that are needed in the sequel. Throughout, 
$\tilde{U}$ represents the convex dual function of $U(\cdot ; \omega)$, defined as

$$
\tilde{U}(y, \omega):=\sup _{0 \leq z \leq H(\omega)}\{U(z, \omega)-y z\}
$$

We observe that the function $\tilde{U}$ is closely related to the Legendre-Fenchel transformation of the convex function $-U(-z)$. It can be easily checked that $\tilde{U}(\cdot ; \omega)$ is convex and differentiable everywhere, for each $\omega$. Furthermore, if we denote the generalized inverse function of $U^{\prime}(\cdot, \omega)$ by

$$
I(y, \omega):=\inf \left\{z \in(0, H(\omega)) \mid U^{\prime}(z, \omega)<y\right\}
$$

with the convention that $\inf \emptyset=\infty$, then it holds that

$$
\tilde{U}^{\prime}(y, \omega)=-(I(y ; \omega) \wedge H), \quad \forall y>0,
$$

and the function $\tilde{U}$ has the following representation:

$$
\tilde{U}(y, w)=U(I(y, w) \wedge H(\omega), \omega)-y(I(y, w) \wedge H(\omega)) .
$$

Remark 3.1. We point out that the random fields defined in (3.1) and (3.2) are $\mathbb{B}([0, \infty)) \times \mathcal{F}_{T^{-}}$ measurable. For instance, in the case of $\tilde{U}$, we can write

$$
\tilde{U}(y, \omega)=\sup _{z \geq 0}\{U(z, \omega)-y z\} 1_{\{z \leq H(\omega)\}}
$$

and we will only need to check that $(y, \omega) \rightarrow\{U(z, \omega)-y z\} 1_{\{z \leq H(\omega)\}}$ is jointly measurable for each fixed $z$. This last fact follows because the random field in question is continuous in the spatial variable $y$ for each $\omega$ and is $\mathcal{F}_{T}$-measurable for each $y$. In light of (3.3), it transpires that the random field $I(y, \omega) \wedge H(\omega)$ is jointly measurable. Given that the subsequent dual problems and corresponding solutions are given in terms of the fields $\tilde{U}(y, \omega)$ and $I(y, \omega) \wedge$ $H(\omega)$ (see Definition 3.3 and Theorem 3.5), the measurability of several key random variable below is guaranteed.

Next, we introduce the traditional dual class (cf. [14]).

Definition 3.2. Let $\widetilde{\Gamma}$ be the class of nonnegative supermartingales $\xi$ such that

(i) $0 \leq \xi(0) \leq 1$,

(ii) for each locally bounded admissible trading strategy $\beta,\left\{\xi(t) V_{t}^{\beta}\right\}_{t \leq T}$ is a supermartingale. 
To motivate the construction of the dual problems below we note that if $\xi \in \widetilde{\Gamma}$ and $V$ is the value process of a self-financing admissible portfolio with initial endowment $V_{0} \leq z$, then $\mathbb{E}\left[\xi(T)\left(V_{T} \wedge H\right)\right] \leq z$, and it follows that

$$
\begin{aligned}
\mathbb{E}\left[U\left(V_{T}, \cdot\right)\right] & \leq \mathbb{E}\left[U\left(V_{T} \wedge H, \cdot\right)\right]-y\left(\mathbb{E}\left[\xi(T)\left(V_{T} \wedge H\right)\right]-z\right) \\
& \leq \mathbb{E}\left\{\sup _{0 \leq z^{\prime} \leq H(\cdot)}\left\{U\left(z^{\prime}, \cdot\right)-y \xi(T) z^{\prime}\right\}\right\}+z y \\
& =\mathbb{E}\{\tilde{U}(y \xi(T), \cdot)\}+z y
\end{aligned}
$$

for any $y \geq 0$. The dual problem is defined as follows.

Definition 3.3. Given a subclass $\Gamma \subset \widetilde{\Gamma}$, the minimization problem

$$
v_{\Gamma}(y):=\inf _{\xi \in \Gamma} \mathbb{E}[\tilde{U}(y \xi(T), \omega)], \quad y>0,
$$

is called the "dual problem induced by $\Gamma . "$ The class $\Gamma$ is referred to as a dual domain (or class) and $v_{\Gamma}(\cdot)$ is called its dual value function.

Notice that, by (3.6) and (3.7), we have the following weak duality relation between the primal and dual value functions:

$$
u(z) \leq v_{\Gamma}(y)+z y
$$

valid for all $z, y \geq 0$. The effectiveness of the dual problem depends on the attainability of the lower bound in (3.8) for some $y^{*}=y^{*}(z)>0$ (in which case, we say that strong duality holds), and the attainability of its corresponding dual problem (3.7). The following well-known properties will be needed for future reference. Their proofs are standard and are outlined in Appendix B for the sake of completeness.

Proposition 3.4. The dual value function $v_{\Gamma}$ corresponding to a subclass $\Gamma$ of $\widetilde{\Gamma}$ satisfies the following properties

(1) $v_{\Gamma}$ is nonincreasing on $(0, \infty)$ and $\mathbb{E}[U(0 ; \cdot)] \leq v_{\Gamma}(y) \leq \mathbb{E}[U(H ; \cdot)]$.

(2) If

$$
0<w_{\Gamma}:=\sup _{\xi \in \Gamma} \mathbb{E}[\xi(T) H]<\infty
$$

then $v_{\Gamma}$ is uniformly continuous on $(0, \infty)$, and

$$
\lim _{y \downarrow 0} \frac{\mathbb{E}[U(H ; \cdot)]-v_{\Gamma}(y)}{y}=\sup _{\xi \in \Gamma} \mathbb{E}[\xi(T) H]
$$


(3) There exists a process $\tilde{\xi} \in \tilde{\Gamma}$ such that $\mathbb{E}[\tilde{U}(y \tilde{\xi}(T), \cdot)] \leq v_{\Gamma}(y)$.

(4) If $\Gamma$ is a convex set, then (i) $v_{\Gamma}$ is convex, and with (ii) there exists a $\xi^{*} \in \widetilde{\Gamma}$ attaining the minimum $v_{\Gamma}(y)$. Furthermore, the optimum $\xi^{*}$ can be "approximated" by elements of $\Gamma$ in the sense that there exists a sequence $\left\{\xi^{n}\right\}_{n} \subset \Gamma$ for which $\xi^{n}(T) \rightarrow \xi^{*}(T)$, a.s.

We now give a result that is crucial for proving the strong duality in (3.8). The result follows from arguments quite similar to those in [9, Theorem 9.3]. For the sake of completeness, we outline the proof in Appendix B.

Theorem 3.5. Suppose that (3.9) is satisfied and $\Gamma$ is convex, then, for any $z \in\left(0, w_{\Gamma}\right)$, there exist $y(z)>0$ and $\xi_{y(z)}^{*} \in \widetilde{\Gamma}$ such that

(i) $\mathbb{E}\left[\tilde{U}\left(y(z) \xi_{y(z)}^{*}(T), \omega\right)\right] \leq \mathbb{E}[\tilde{U}(y(z) \xi(T), \omega)]$, for all $\xi \in \Gamma$;

(ii) $\mathbb{E}\left[V_{z}^{\Gamma} \xi_{y(z)}^{*}(T)\right]=z$, where

$$
V_{z}^{\Gamma}:=I\left(y(z) \xi_{y(z)}^{*}(T)\right) \wedge H
$$

(iii) $u(z) \leq \mathbb{E}\left[U\left(V_{z}^{\Gamma} ; \omega\right)\right]$.

We note that Theorem 3.5 provides essentially an upper bound for the optimal final utility of the form $\mathbb{E}\left[U\left(V_{z}^{\Gamma} ; \omega\right)\right]$, for certain "reduced" contingent claim $V_{z}^{\Gamma} \leq H$. By suitably choosing the dual class $\Gamma$, we shall prove in the next two sections that this reduced contingent claim is (super-) replicable with an initial endowment $z$.

\section{Characterization of the Optimal Dual}

We now give a full description of a dual class $\Gamma$ for which strong duality, that is, $u(z)=$ $v_{\Gamma}(y)+z y$, holds. Denote $\mho^{+}$to be the class of all real-valued càdlàg, nondecreasing, adapted processes $A$ null at zero. We will call such a process "increasing." In what follows we let $\mathcal{\varepsilon}(X)$ be the Doléans-Dade stochastic exponential of the semimartingale X (see, e.g., [17] for their properties). Let

$$
\mathcal{S}:=\left\{X_{t}:=\int_{0}^{t} G(s) d W_{s}+\int_{0}^{t} \int_{\mathbb{R}_{0}} F(s, z) \widetilde{N}(d s, d z): F \geq-1\right\},
$$

and consider the associated class of exponential local supermartingales:

$$
\Gamma(\mathcal{S}):=\left\{\xi:=\xi_{0} \mathcal{E}(X-A): X \in \mathcal{S}, A \text { increasing, and } \xi \geq 0\right\}
$$

In (4.1), we assume that $G \in L_{\mathrm{loc}}^{2}(W), F \in G_{\mathrm{loc}}(N)$, and that $F(t, \cdot)=G(t)=0$, for all $t \geq T$. The following result shows not only that the class

$$
\Gamma:=\widetilde{\Gamma} \cap \Gamma(\mathcal{S})
$$


is convex, but also that the dual optimum, whose existence is guaranteed from Theorem 3.5, remains in $\Gamma$. The proof of this result is based on a powerful representation for nonnegative supermartingales due to Föllmer and Kramkov [12] (see Theorem A.1 in Appendix A), and a technical result about the closedness of the class of integrals with respect to Poisson random measures, under Émery's topology. We shall defer the presentation of these two fundamental results to Appendix A in order to continue with our discussion of the dual problem.

Theorem 4.1. The class $\Gamma$ is convex, and if (3.9) is satisfied, the dual optimum $\xi_{y(z)}^{*}$ of Theorem 3.5 belongs to $\Gamma$, for any $0<z<w_{\Gamma}$.

Proof. Let us check that $\mathcal{S}$ meets with the conditions in Theorem A.1. Indeed, each $X$ in $\mathcal{S}$ is locally bounded from below since, defining $\tau_{n}:=\inf \left\{t \geq 0: X_{t}<-n\right\}$,

$$
X_{t}^{\tau_{n}} \geq X_{\tau_{n}^{-}}-\left(\Delta X_{\tau_{n}}\right)^{-} 1_{\tau_{n}<\infty} \geq-n-1,
$$

where $(x)^{-}=-x 1_{x<0}$. Condition (i) of Theorem A.1 is straightforward, while condition (ii) follows from Theorem A.3. Finally, condition (iii) holds because the processes in $\mathcal{S}$ are already local martingales with respect to $\mathbb{P}$ and hence $\mathbb{P} \in D(\mathcal{S})$ with $A^{\mathcal{S}}(\mathbb{P}) \equiv 0$. By Proposition A.2 we conclude that $\Gamma(\mathcal{S})$ is convex and closed under Fatou convergence on dense countable sets. On the other hand, $\widetilde{\Gamma}$ is also convex and closed under Fatou convergence, and thus so is the class $\Gamma:=\widetilde{\Gamma} \cap \Gamma(\mathcal{S})$. To check the second statement, recall that the existence of the dual minimizer $\xi_{y(z)}^{*}$ in Theorem 3.5 is guaranteed from Proposition 3.4, where it is seen that $\xi_{y(z)}^{*}$ is the Fatou limit of a sequence in $\Gamma$ (see the proof of Proposition 3.4). This suffices to conclude that $\xi_{y(z)}^{*} \in \Gamma$ since $\Gamma$ is closed under under Fatou convergence.

In the rest of this section, we present some properties of the elements in $\Gamma$ and of the dual optimum $\xi^{*} \in \Gamma$. In particular, conditions on the "parameters" $(G, F, A)$ so that $\xi \in$ $\Gamma(\mathcal{S})$ is in $\widetilde{\Gamma}$ are established. First, we note that without loss of generality, $A$ can be assumed predictable.

Lemma 4.2. Let

$$
\xi:=\xi_{0} \mathcal{\varepsilon}(X-A) \in \Gamma(\mathcal{S}) .
$$

Then, there exist a predictable process $A^{p} \in \mathcal{U}^{+}$and a process $\hat{X} \in \mathcal{S}$ such that $\xi=\xi_{0} \mathcal{E}\left(\hat{X}-A^{p}\right)$.

Proof. Let $X_{t}:=\int_{0}^{t} G(s) d W_{s}+\int_{0}^{t} \int_{\mathbb{R}_{0}} F(s, z) \widetilde{N}(d s, d z) \in \mathcal{S}$. Since $F \in G_{\text {loc }}(N)$, there are stopping times $\tau_{n}^{\prime} \nearrow \infty$ such that

$$
\mathbb{E} \int_{0}^{\tau_{n}^{\prime}} \int_{\mathbb{R}}|F(s, z)| 1_{|F|>1} v(d z) d s<\infty
$$

Compare with [17, Theorem II.1.33]. Now, define

$$
\tau_{n}^{\prime \prime}:=\inf \left\{t \geq 0: A_{t}>n\right\},
$$


and $\tau_{n}:=\tau_{n}^{\prime} \wedge \tau_{n}^{\prime \prime}$. Then,

$$
\begin{aligned}
\mathbb{E} A_{\infty}^{\tau_{n}} & =\mathbb{E}\left[A_{\tau_{n}^{-}}\right]+\mathbb{E}\left[\Delta A_{\tau_{n}}\right] \leq n+1+\mathbb{E}\left[\left|F\left(\tau_{n}, Z_{\tau_{n}}\right)\right|\right] \\
& \leq n+2+\mathbb{E} \int_{0}^{\tau_{n}} \int_{\mathbb{R}}|F(s, z)| 1_{|F|>1} v(d z) d s<\infty,
\end{aligned}
$$

where we used that $\Delta X_{t}-\Delta A_{t} \geq-1$. Therefore, $A$ is locally integrable, increasing, and thus, its predictable compensator $A^{p}$ exists. Now, by the representation theorem for local martingales (see [17, Theorem III.4.34]), the local martingale $X^{\prime}:=A-A^{p}$ admits the representation

$$
X^{\prime}:=\int_{0} G^{\prime}(s) d W_{s}+\int_{0} \int_{\mathbb{R}_{0}} F^{\prime}(s, z) \widetilde{N}(d s, d z) .
$$

Finally, $\xi=\xi_{0} \varepsilon(X-A)=\xi_{0} \varepsilon\left(X-X^{\prime}-A^{p}\right)$. The conclusion of the proposition follows since $\widehat{X}:=X-X^{\prime}$ is necessarily in $\mathcal{S}$.

The following result gives necessary conditions for a process $\xi \in \Gamma(\mathcal{S})$ to belong to $\widetilde{\Gamma}$. Recall that a predictable increasing process $A$ can be uniquely decomposed as the sum of three predictable increasing processes,

$$
A=A^{c}+A^{s}+A^{d},
$$

where $A^{c}$ is the absolutely continuous part, $A^{s}$ is the singular continuous part, and $A_{t}^{d}=$ $\sum_{s \leq t} \Delta A_{s}$ is the jump part (cf. [18, Theorem 19.61]).

Proposition 4.3. Let $\xi:=\xi_{0} \varepsilon(X-A) \geq 0$, where $\xi_{0}>0$,

$$
X_{t}:=\int_{0}^{t} G(s) d W_{s}+\int_{0}^{t} \int_{\mathbb{R}_{0}} F(s, z) \widetilde{N}(d s, d z) \in \mathcal{S},
$$

and $A$ is an increasing predictable process. Let $\tau$ be the "sinking time" of the supermartingale $\xi$ :

$$
\tau:=\sup _{n} \inf \left\{t: \xi_{t}<\frac{1}{n}\right\}=\inf \left\{t: \Delta X_{t}=-1 \text { or } \Delta A_{t}=1\right\} \text {. }
$$

Also, let $a_{t}=d A_{t}^{c} / d t$. Then, $\left\{\xi_{t} S_{t}\right\}_{t \leq T}$ is a supermartingale if and only if the following two conditions are satisfied.

(i) There exist stopping times $\tau_{n} \nearrow \tau$ such that

$$
\mathbb{E} \int_{0}^{\tau_{n}} \int_{\mathbb{R}}|v(s, z) F(s, z)| v(d z) d s<\infty .
$$

(ii) For $\mathbb{P}$-a.e. $\omega \in \Omega$,

$$
h_{t} \leq a_{t},
$$


for almost every $t \in[0, \tau(\omega)]$, where

$$
h_{t}:=b_{t}+\sigma_{t} G(t)+\int_{\mathbb{R}} v(t, z) F(t, z) v(d z)
$$

Proof. Recall that $\xi$ and $S$ satisfy the SDE's

$$
\begin{aligned}
& d \xi_{t}=\xi_{t^{-}}\left(G(t) d W_{t}+\int_{\mathbb{R}_{0}} F(t, z) \widetilde{N}(d t, d z)-d A_{t}\right) \\
& d S_{t}=S_{t^{-}}\left(b_{t} d t+\sigma_{t} d W_{t}+\int_{\mathbb{R}_{0}} v(t, z) \widetilde{N}(d t, d z)\right) .
\end{aligned}
$$

Integration by parts and the predictability of $A$ yield that

$$
\begin{aligned}
\xi_{t} S_{t}= & \text { local martingale }+\int_{0}^{t} b_{s} \xi_{s^{-}} S_{s^{-}} d s+\int_{0}^{t} \sigma_{s} G(s) \xi_{s^{-}} S_{s^{-}} d s \\
& -\int_{0}^{t} \xi_{s^{-}} S_{s^{-}} d A_{s}+\int_{0}^{t} \int_{\mathbb{R}_{0}} v(s, z) F(s, z) \xi_{s^{-}} S_{s^{-}} N(d s, d z) .
\end{aligned}
$$

Suppose that $\left\{\xi_{t} S_{t}\right\}_{t \geq 0}$ is a nonnegative supermartingale. Then, the integral $\int_{0}^{t} \int_{\mathbb{R}^{\prime}} v(s, z) F(s, z) \xi_{s^{-}} S_{s^{-}} N(d s, d z)$ must have locally integrable variation in light of the Doob-Meyer decomposition for supermartingale (see, e.g., [16, Theorem III.13]). Therefore, there exist stopping times $\tau_{n}^{1} \nearrow \infty$ such that

$$
\mathbb{E} \int_{0}^{\tau_{n}^{1}} \int_{\mathbb{R}}\left|v(s, z) F(s, z) \xi_{s^{-}} S_{s^{-}}\right| v(d z) d s<\infty
$$

Then, (i) is satisfied with $\tau_{n}:=\tau_{n}^{1} \wedge \tau_{n}^{2} \wedge \tau_{n}^{3}$, where $\tau_{n}^{2}:=\inf \left\{t: \xi_{t}<1 / n\right\}$ and $\tau_{n}^{3}:=\inf \left\{t: \widetilde{S}_{t}<\right.$ $1 / n\}$. Next, we can write (4.17) as

$$
\xi_{t} S_{t}=\text { local martingale }-\int_{0}^{t} \xi_{s^{-}} S_{s^{-}}\left(d A_{s}-h_{s} d s\right)
$$

By the Doob-Meyer representation for supermartingales and the uniqueness of the canonical decomposition for special semimartingales, the last integral must be increasing. Then, $a_{t} \geq h_{t}$ for $t \leq \tau$ since $\xi_{t^{-}}>0$ and $\xi_{t}=0$ for $t \geq \tau$ (see [17, Theorem I.4.61]). bounded,

We now turn to the sufficiency of conditions (i) and (ii). Since $\left\{\xi_{t^{-}} S_{t^{-}}\right\}_{t \geq 0}$ is locally

$$
\int_{0}^{t} \int_{\mathbb{R}}|v(s, z) F(s, z)| \xi_{s^{-}} S_{s^{-}} 1_{s \leq \tau_{n}} v(d z) d s
$$


is locally integrable. Then, from (4.17), we can write

$$
\xi_{t \wedge \tau_{n}} S_{t \wedge \tau_{n}}=\text { local martingale }-\int_{0}^{t} \xi_{s^{-}} S_{s^{-}} 1_{s \leq \tau_{n}}\left(d A_{s}-h_{s} d s\right)
$$

Condition (ii) implies that $\left\{\xi_{t \wedge \tau_{n}} S_{t \wedge \tau_{n}}\right\}$ is a supermartingale, and by Fatou, $\left\{\xi_{t \wedge \tau} S_{t \wedge \tau}\right\}_{t \geq 0}$ will be a supermartingale. This concludes the prove since $\xi_{t}=0$ for $t \geq \tau$, and thus, $\xi_{t \wedge \tau} S_{t \wedge \tau}=\xi_{t} S_{t}$, for all $t \geq 0$.

The following result gives sufficient and necessary conditions for $\xi \in \Gamma(\mathcal{S})$ to belong to $\widetilde{\Gamma}$. Its proof is similar to that of Proposition 4.3.

Proposition 4.4. Under the setting and notation of Proposition $4.3, \xi \in \Gamma(\mathcal{S})$ belongs to $\widetilde{\Gamma}$ if and only if condition (i) in Proposition 4.3 holds and, for any locally bounded admissible trading strategies $\beta$,

$$
\mathbb{P}\left[\left\{\omega: h_{t} \beta_{t} \leq a_{t}, \text { for a.e. } t \in[0, \tau(\omega)]\right\}\right]=1 .
$$

The previous result can actually be made more explicit under additional information on the structure of the jumps exhibited by the stock price process. We consider two cases: when the jumps come from the superposition of shot-noise Poisson processes, and when the random field $v$ exhibit a multiplicative structure. Let us first extend Proposition 2.2 in these two cases.

Proposition 4.5. (i) Suppose that $v$ is atomic with finitely many atoms $\left\{z_{i}\right\}_{i=1}^{k}$, then, a predictable locally bounded strategy $\beta$ is admissible if and only if $\mathbb{P} \times d t$-a.e.

$$
-\frac{1}{\max _{i} v\left(t, z_{i}\right) \vee 0} \leq \beta_{t} \leq-\frac{1}{\min _{i} v\left(t, z_{i}\right) \wedge 0} .
$$

(ii) Suppose that $v(t, z)=\zeta_{t} \vartheta(z)$, for a predictable locally bounded process $\zeta$ such that $\mathbb{P} \times d t$ a.e. $\zeta_{t}(\omega) \neq 0$ and $\zeta_{t}^{-1}$ is locally bounded, and a deterministic function $\vartheta$ such that $v(\{z: \vartheta(z)=0\})=$ 0 , then, a predictable locally bounded strategy $\beta$ is admissible if and only if $\mathbb{P} \times d t$-a.e.

$$
-\frac{1}{\bar{\vartheta} \vee 0} \leq \beta_{t} \zeta_{t} \leq-\frac{1}{\underline{\vartheta} \wedge 0}
$$

where $\bar{\vartheta}:=\sup \{\vartheta(z): z \in \operatorname{supp}(v)\}$ and $\underline{\vartheta}:=\inf \{\vartheta(z): z \in \operatorname{supp}(v)\}$.

Proof. From Proposition 2.2, recall that $\mathbb{P}$-a.s.

$$
\beta_{t} v\left(t, \Delta Z_{t}\right) \geq-1
$$

for a.e. $t \leq T$. Then, for any closed set $C \subset \mathbb{R}_{0}, 0 \leq s<t$, and $A \in \mathcal{F}_{s}$,

$$
\sum_{s<u \leq t} X_{A}(\omega) X_{C}\left(\Delta Z_{u}\right)\left\{\beta_{u} v\left(u, \Delta Z_{u}\right)+1\right\} \geq 0
$$


Taking expectation, we get

$$
\mathbb{E} \int_{S}^{t} X_{A} \int_{C}\left\{\beta_{u} v(u, z)+1\right\} v(d z) d u \geq 0
$$

Since such processes $H_{u}(\omega):=\chi_{A \times(s, t]}(\omega, u)$ generate the class of predictable processes, we conclude that $\mathbb{P} \times d t$-a.e.

$$
-1 \leq \beta_{t} \frac{\int_{C} v(t, z) \mathcal{v}(d z)}{v(C)}
$$

Let us prove (ii) (the proof of (i) is similar). Notice that

$$
\inf _{z \in U} \vartheta(z)=\inf _{C \subset \mathbb{R}_{0}} \frac{\int_{C} \vartheta(z) v(d z)}{v(C)} \leq \sup _{C \subset \mathbb{R}_{0}} \frac{\int_{C} \vartheta(z) v(d z)}{v(C)}=\sup _{z \in U} \vartheta(z)
$$

where $U$ is the support of $\mathcal{v}$. Suppose that $\inf _{z \in U} \vartheta(z)<0<\sup _{z \in U} \vartheta(z)$. Then, by considering closed sets $C_{n}, C_{n}^{\prime} \subset \mathbb{R}_{0}$ such that

$$
\frac{\int_{C_{n}} \vartheta(z) v(d z)}{v\left(C_{n}\right)} \nearrow \sup _{z} \vartheta(z), \quad \frac{\int_{C_{n}^{\prime}} \vartheta(z) v(d z)}{v\left(C_{n}^{\prime}\right)} \searrow \inf _{z} \vartheta(z),
$$

as $n \rightarrow \infty$, we can prove the necessity. The other two cases (namely, $\inf _{z} \vartheta(z) \geq 0$ or $0 \geq$ $\left.\sup _{z} \vartheta(z)\right)$ are proved in a similar way. Sufficiency follows since, $\mathbb{P}$-a.s.,

$$
\begin{aligned}
\left\{t \leq T: \beta_{t} \zeta_{t} v\left(\Delta Z_{t}\right)<-1\right\} & \subset\left\{t \leq T: \beta_{t} \zeta_{t} \sup _{z \in U} v(z)<-1\right\} \\
& \cup\left\{t \leq T: \beta_{t} \zeta_{t} \inf _{z \in U} v(z)<-1\right\} .
\end{aligned}
$$

Example 4.6. It is worth pointing out some consequences

(a) In the time homogeneous case, where $v(t, z)=z$, the extreme points of the support of $v$ (or what accounts to the same, the infimum and supremum of all possible jump sizes) determine completely the admissible strategies. For instance, if the Lévy process can exhibit arbitrarily large or arbitrarily close to -1 jump sizes, then

$$
0 \leq \beta_{t} \leq 1
$$

a constraint that can be interpreted as absence of shortselling and bank borrowing (this fact was already pointed out by Hurd [19]).

(b) In the case that $\underline{\vartheta} \geq 0$, the admissibility condition takes the form $-1 / \bar{\vartheta} \leq \beta_{t} \zeta_{t}$. If in addition $\zeta .<0$ (such that the stock prices exhibit only downward sudden 
movements), then $-1 /\left(\bar{\vartheta} \zeta_{t}\right) \geq \beta_{t}$, and $\beta$. $\equiv-c$, with $c>0$ arbitrary, is admissible. In particular, from Proposition 4.4, if $\xi \in \Gamma(\mathcal{S})$ belongs to $\widetilde{\Gamma}$, then a.s. $h_{t} \beta_{t} \leq$ $a_{t}$, for a.e. $t \leq \tau$. This means that $\xi \in \Gamma(\mathcal{S}) \cap \tilde{\Gamma}$ if and only if condition (i) in Proposition 4.3 holds and $\mathbb{P}$-a.s. $h_{t} \geq 0$, for a.e. $t \leq \tau$. For a general $\zeta$ and still assuming that $\underline{\vartheta} \geq 0$, it follows that $\beta$ is admissible and $\xi \in \Gamma(\mathcal{S}) \cap \tilde{\Gamma}$ satisfy that $\mathbb{P}$-a.s.

$$
-\frac{1}{\bar{\vartheta}\left(\zeta_{t} \vee 0\right)} \leq \beta_{t} \leq-\frac{1}{\bar{\vartheta}\left(\zeta_{t} \wedge 0\right)}, \quad h_{t} \zeta_{t}^{-1} 1_{\{t \leq \tau\}} \leq 0
$$

for a.e. $t \geq 0$.

We now extend Proposition 4.4 in the two cases introduced in Proposition 4.5. Its proof follows from Propositions 4.4 and 4.5 .

Proposition 4.7. Suppose that either (i) or (ii) in Proposition 4.5 is satisfied, in which case, define

$$
\widehat{h}_{t}:= \begin{cases}-\frac{h_{t}}{\max _{i} v\left(t, z_{i}\right) \vee 0} 1_{\left\{h_{t}<0\right\}}-\frac{h_{t}}{\min _{i} v\left(t, z_{i}\right) \wedge 0} 1_{\left\{h_{t}>0\right\}} & \text { if (i) holds true, } \\ -\frac{h_{t} \zeta_{t}^{-1}}{\bar{\vartheta} \vee 0} 1_{\left\{h_{t} \zeta_{t}^{-1}<0\right\}}-\frac{h_{t} \zeta_{t}^{-1}}{\underline{\vartheta} \wedge 0} 1_{\left\{h_{t} \zeta_{t}^{-1}>0\right\}} & \text { if (ii) hold strue, }\end{cases}
$$

then, a process $\xi \in \Gamma(\mathcal{S})$ belongs to $\widetilde{\Gamma}$ if and only if condition (i) in Proposition 4.3 holds, and for $\mathbb{P}$-a.e. $\omega, \widehat{h}_{t}(\omega) 1_{\{t \leq \tau(\omega)\}} \leq a_{t}(\omega) 1_{\{t \leq \tau(\omega)\}}$, for a.e. $t \geq 0$.

We remark that the cases $\underline{\vartheta} \geq 0$ and $\bar{\vartheta} \leq 0$ do not lead to any absurd in the definition of $\widehat{h}$ above as we are using the convention that $0 \cdot \infty=0$. Indeed, for instance, if $\underline{\vartheta} \geq 0$, it was seeing that $h_{t} \zeta_{t}^{-1} \leq 0$, for a.e. $t \leq \tau$, and thus, we set the second term in the definition of $\widehat{h}$ to be zero.

Now we can give a more explicit characterization of the dual solution $\xi^{*}=\varepsilon\left(X^{*}-\right.$ $\left.A^{*}\right)$ to problem (3.7), whose existence was established in Theorem 4.1. For instance, we will see that $A^{*}$ is absolutely continuous up to a predictable stopping time. Below, we refer to Proposition 4.3 for the notation.

Proposition 4.8. Let $\xi:=\xi_{0} \mathcal{E}(X-A) \in \Gamma(\mathcal{S}), \tau_{A}:=\inf \left\{t: \Delta A_{t}=1\right\}$, and $\tilde{A}_{t}:=\int_{0}^{t} a_{S} d s+1_{\left\{t \geq \tau_{A}\right\}}$. The followings two statements hold true.

(1) $\tilde{\xi}:=\xi_{0} \varepsilon(X-\tilde{A}) \geq \xi$. Furthermore, $\xi \in \widetilde{\Gamma}$ if and only if $\tilde{\xi} \in \widetilde{\Gamma}$.

(2) Suppose that either of the two conditions in Proposition 4.7 are satisfied and denote

$$
\widehat{A}_{t}:=\int_{0}^{t} \widehat{h}_{s} 1_{s \leq \tau} d s+1_{\left\{t \geq \tau_{A}\right\}}
$$

where $\widehat{h}$ is defined accordingly to the assumed case. Then, $\xi . \leq \widehat{\xi}$, and furthermore, the process $\widehat{\xi}:=\xi_{0} \varepsilon(X-\widehat{A})$ belongs to $\widetilde{\Gamma}$ if $\xi \in \widetilde{\Gamma}$. 
Proof. Let $A^{c}, A^{s}, A^{d}$ denote the increasing predictable processes in decomposition (4.10) of $A$. Since $A$ is predictable, there is no common jump times between $X$ and $A$. Then,

$$
\begin{aligned}
\xi_{t} & =\xi_{0} e^{X_{t}-A_{t}-(1 / 2)\left\langle X^{c}, X^{c}\right\rangle_{t}} \prod_{s \leq t}\left(1+\Delta X_{s}\right) e^{-\Delta X_{s}} \prod_{s \leq t}\left(1-\Delta A_{s}\right) e^{\Delta A_{s}} \\
& \leq \xi_{0} e^{X_{t}-A_{t}^{c}-(1 / 2)\left\langle X^{c}, X^{c}\right\rangle_{t}} \prod_{s \leq t}\left(1+\Delta X_{S}\right) e^{-\Delta X_{s}} 1_{\left\{t<\tau_{A}\right\}}=\widetilde{\xi}_{t},
\end{aligned}
$$

where we used that $A_{t}-\sum_{s \leq t} \Delta A_{s}=A_{t}^{c}+A_{t}^{s} \geq A_{t}^{c}$, and $\prod_{s \leq t}\left(1-\Delta A_{s}\right) \leq 1_{\left\{t<\tau_{A}\right\}}$. Since both processes $\xi$ and $\tilde{\xi}$ enjoy the same absolutely continuous part, and the same sinking time, the second statement in (1) is straightforward from Proposition 4.4. Part (2) follows from Proposition 4.7 since the process $\widehat{a}_{t}:=\widehat{h}_{t} 1_{t \leq \tau}$ is nonnegative, predictable (since $h$ is predictable), and locally integrable (since $0 \leq \widehat{h} \leq a$ ).

We remark that part (2) in Proposition 4.8 remains true if we take $\widehat{A}_{t}:=\int_{0}^{t} \widehat{h}_{s} 1_{s \leq \tau_{A}} d s+$ $1_{\left\{t \geq \tau_{A}\right\}}$. The following result is similar to Proposition 3.4 in $\mathrm{Xu}$ [5] and implies, in particular, that the optimum dual $\xi^{*}$ can be taken to be a local martingale.

Proposition 4.9. Suppose that either (i) or (ii) of Proposition 4.5 is satisfied. Moreover, in the case of condition (ii), assume additionally that

$$
v(\{z \in \operatorname{supp}(v) \backslash\{0\}: \vartheta(z)=c\})>0,
$$

for $c=\underline{\vartheta}$ if $\bar{\vartheta}>0$, and for $c=\bar{\vartheta}$ if $\underline{\vartheta}<0$. Let $\xi \in \tilde{\Gamma} \cap \Gamma(\mathcal{S})$. Then, there exists $\tilde{X} \in \mathcal{S}$ such that $\tilde{\xi}:=\xi_{0} \varepsilon(\tilde{X}) \in \tilde{\Gamma}$ and $\xi . \leq \tilde{\xi}$. Furthermore, $\left\{\tilde{\xi}(t) V_{t}^{\beta}\right\}_{t \leq T}$ is a local martingale for all locally bounded admissible trading strategies $\beta$.

Proof. Let us prove the case when condition (i) in Proposition 4.5 is in force. In light of Proposition 4.8, we assume without loss of generality that $A_{t}=\int_{0}^{t} a_{t} d t+1_{\left\{t \geq \tau_{A}\right\}}$, with $a_{t}:=$ $\widehat{h}_{t} 1_{\{t \leq \tau\}}$. Assume that $\min _{i} v\left(t, z_{i}\right)<0<\max _{i} v\left(t, z_{i}\right)$. Otherwise if, for instance, $\max _{i} v\left(t, z_{i}\right) \leq$ 0 , then it can be shown that $h_{t} \geq 0$, a.s. (similarly to case (b) in Example 4.6), and the first term of $\widehat{h}$ is 0 under our convention that $\infty \cdot 0=0$. Notice that, in any case, one can find a predictable process $z$ taking values on $\left\{z_{i}\right\}_{i=1}^{n}$, such that

$$
\widehat{h}_{t}=-\frac{h_{t}}{v(t, z(t))} .
$$

Write $\tilde{X}$ : : $=\int_{0} G(s) d W_{s}+\int_{0} \int_{\mathbb{R}_{0}} \tilde{F}(s, z) d \widetilde{N}(s, z)$ for an $\widetilde{F} \in G_{\text {loc }}(N)$ to be determined in the sequel. For $\widetilde{\xi} \geq \xi$ it suffices to prove the existence of a field $D$ satisfying both conditions below:

$$
\text { (a) } D \geq 0, \quad \text { (b) } \int_{\mathbb{R}_{0}} D(t, z) v(d z) 1_{\{t \leq \tau\}} \leq \widehat{h}_{t} 1_{\{t \leq \tau\}} \text {, }
$$


(then, $\widetilde{F}$ is defined as $D+F$ ). Similarly, for $\widehat{\xi}$ to belong to $\widetilde{\Gamma}$ it suffices that

$$
\text { (c) } h_{t}+\int_{\mathbb{R}_{0}} v(t, z) D(t, z) v(d z)=0
$$

Taking

$$
D(t, z):=-\frac{h_{t}}{v(t, z(t)) \mathcal{v}(\{z(t)\})} 1_{\{z=z(t)\}},
$$

clearly nonnegative, (b) and (c) hold with equality. Moreover, the fact that inequalities (c) hold with equality implies that $\left\{\widehat{\xi}(t) V_{t}^{\beta}\right\}_{t \leq T}$ is a local martingale for all locally bounded admissible trading strategy $\beta$ (this can be proved using the same arguments as in the sufficiency part of Proposition 4.3). Now suppose that condition (ii) in Proposition 4.5 holds. For simplicity, let us assume that $\underline{\vartheta}<0<\bar{\vartheta}$ (the other cases can be analyzed following arguments similar to Example 4.6). Notice that (4.37) implies the existence of a Borel $\underline{C}$ (resp., $\bar{C})$ such that $\vartheta(z) \equiv \underline{\vartheta}$ on $\underline{C}(\operatorname{resp} ., \vartheta(z) \equiv \bar{\vartheta}$ on $\bar{C}$ ) and $0<v(\underline{C}), v(\bar{C})<\infty$. Taking

$$
D(t, z):=-\frac{h_{t} \zeta_{t}^{-1}}{\bar{\vartheta} v(\bar{C})} 1_{\bar{C}}(z) 1_{\left\{h_{t} \zeta_{t}^{-1}<0\right\}}-\frac{h_{t} \zeta_{t}^{-1}}{\underline{\vartheta} \underline{v}(\underline{C})} 1_{\underline{C}}(z) 1_{\left\{h_{t} \zeta_{t}^{-1}>0\right\}},
$$

(b) and (c) above will hold with equality.

\section{Replicability of the Upper Bound}

We now show that the tentative optimum final wealth $V_{z}^{\Gamma}$, suggested by the inequality (iii) in Theorem 3.5, is (super-) replicable. We will combine the dual optimality of $\xi^{*}$ with the superhedging theorem, which states that given a contingent claim $\widehat{H}$ satisfying $\bar{w}:=\sup _{\mathbb{Q} \in \mathcal{M}} \mathbb{E}_{\mathbb{Q}}\{\widehat{H}\}<$ $\infty$, one can find for any fixed $z \geq \bar{w}$ an admissible trading strategy $\beta^{*}$ (depending on $z$ ) such that $V_{T}^{z, \beta^{*}} \geq \widehat{H}$ almost surely (see Kramkov [2], and also Delbaen and Schachermayer [1]). Recall that $\mathcal{M}$ denotes the class of all equivalent risk neutral probability measures.

Proposition 5.1. Under the setting and conditions of Theorem 3.5, for any $0<z<w_{\Gamma}$, there is an admissible trading strategy $\beta^{*}$ for $z$ such that

$$
V_{T}^{z, \beta^{*}} \geq I\left(y(z) \xi_{y(z)}^{*}(T)\right) \wedge H
$$

and thus, the optimum of $u(z)$ is reached at the strategy $\beta^{*}$. In particular,

$$
V_{T}^{z, \beta^{*}}=I\left(y(z) \xi_{y(z)}^{*}(T)\right)
$$

when $I\left(y(z) \xi_{y(z)}^{*}(T)\right)<H$. 
Proof. For simplicity, we write $\xi_{t}^{*}:=\xi_{y(z)}^{*}(t), y=y(z)$, and

$$
V^{*}=I\left(y(z) \xi_{y(z)}^{*}(T)\right) \wedge H
$$

Fix an equivalent risk neutral probability measure $\mathbb{Q} \in \mathcal{M}$, and let $\xi_{t}^{\prime}=\left(\left.d \mathbb{Q}\right|_{\bar{f}_{t}} /\left.d \mathbb{P}\right|_{\mathcal{F}_{t}}\right)$ be its corresponding density processes. Here, $\left.\mathbb{Q}\right|_{\mathcal{F}_{t}}$ (resp., $\left.\mathbb{P}\right|_{\mathcal{F}_{t}}$ ) is the restriction of the measure $\mathbb{Q}$ (resp., $\mathbb{P}$ ) to the filtration $\mathcal{F}_{t}$. Under $\mathbb{Q}, S$. is a local martingale, and then, for any locally bounded $\beta, V^{\beta}$ is a $\mathbb{Q}$-local martingale. By [17, Proposition III.3.8.c], $\xi^{\prime} V^{\beta}$ is a $\mathbb{P}_{-}$ local martingale (necessarily nonnegative by admissibility), and thus, $\xi^{\prime}$ is in $\tilde{\Gamma}$. On the other hand, $\xi^{\prime}$ belongs to $\Gamma(\mathcal{S})$ due to the exponential representation for positive local martingales in Kunita [11] (alternatively, by invoking [17, Theorems III.8.3, I.4.34c, and III.4.34], $\xi^{\prime} \in \Gamma(\mathcal{S})$ even if $Z$ were just an additive process $Z$ ). By the convexity of the dual class $\Gamma=\Gamma(\mathcal{S}) \cap \widetilde{\Gamma}$ and the fact that $\xi^{*} \in \Gamma$ (see Theorem 4.1), $\xi^{(\varepsilon)}:=\varepsilon \xi^{\prime}+(1-\varepsilon) \xi^{*}$ belongs to $\Gamma$, for any $0 \leq \varepsilon \leq 1$. Moreover, since $\tilde{U}$ is convex and $\tilde{U}^{\prime}(y)=-(I(y) \wedge H)$,

$$
\left|\frac{\tilde{U}\left(y \xi_{T}^{(\varepsilon)}\right)-\tilde{U}\left(y \xi_{T}^{*}\right)}{\varepsilon}\right| \leq y H\left|\xi_{T}^{\prime}-\xi_{T}^{*}\right| \leq y H\left(\xi_{T}^{\prime}+\xi_{T}^{*}\right) .
$$

The random variable $y H\left(\xi_{T}^{\prime}+\xi_{T}^{*}\right)$ is integrable since by assumption $w_{\Gamma}<\infty$. We can then apply dominated convergence theorem to get

$$
\lim _{\varepsilon \downarrow 0} \frac{1}{\varepsilon}\left\{\mathbb{E}\left[\tilde{U}\left(y \xi_{T}^{(\varepsilon)}\right)\right]-\mathbb{E}\left[\tilde{U}\left(y \xi_{T}^{*}\right)\right]\right\}=-y \mathbb{E}\left[V^{*}\left(\xi_{T}^{\prime}-\xi_{T}^{*}\right)\right]
$$

which is nonnegative by condition (i) in Theorem 3.5. Then, using condition (ii) in Theorem 3.5,

$$
\mathbb{E}_{\mathbb{Q}}\left[V^{*}\right]=\mathbb{E}\left[V^{*} \xi_{T}^{\prime}\right] \leq \mathbb{E}\left[V^{*} \xi_{T}^{*}\right]=z
$$

Since $\mathbb{Q} \in \mathcal{M}$ is arbitrary, $\sup _{\mathbb{Q} \in \mathcal{M}} \mathbb{E}_{\mathbb{Q}}\left[V^{*}\right] \leq z$. By the super-hedging theorem, there is an admissible trading strategy $\beta^{*}$ for $z$ such that

$$
V_{T}^{z, \beta^{*}} \geq I\left(y(z) \xi_{y(z)}^{*}(T)\right) \wedge H
$$

The second statement of the theorem is straightforward since $U(z)$ is strictly increasing on $z<H$. 


\section{Concluding Remarks}

We conclude the paper with the following remarks.

\section{(i) The dual class $\Gamma$}

The dual domain of the dual problem can be taken to be the more familiar class of equivalent risk-neutral probability measures $\mathcal{M}$. To be more precise, define

$$
\bar{\Gamma}:=\left\{\xi_{t}:=\frac{\left.d \mathbb{Q}\right|_{\mathcal{F}_{t}}}{\left.d \mathbb{P}\right|_{\mathcal{F}_{t}}}: \mathbb{Q} \in \mathcal{M}\right\} .
$$

Since $\bar{\Gamma}$ is obviously a convex subclass of $\widetilde{\Gamma}$, Theorem 3.5 implies that, as far as

$$
0<\bar{w}:=\sup _{\xi \in \bar{\Gamma}} \mathbb{E}\left[\xi_{T} H\right]<\infty,
$$

for each $z \in(0, \bar{w})$, there exist $y:=y(z)>0$ and $\xi^{*}:=\xi_{y(z)}^{*} \in \tilde{\Gamma}$ (not necessarily belonging to $\bar{\Gamma}$ ) such that (i)-(iii) in Theorem 3.5 hold with $\Gamma=\bar{\Gamma}$. Finally, one can slightly modify the proof of Proposition 5.1, to conclude the replicability of

$$
V_{z}^{\bar{\Gamma}}:=I\left(y \xi_{T}^{*}\right) \wedge H
$$

Indeed, in the notation of the proof of the Proposition 5.1, the only step which needs to be justified in more detail is that

$$
\mathbb{E}\left[\tilde{U}\left(y \xi_{T}^{*}\right)\right] \leq \mathbb{E}\left[\tilde{U}\left(y \xi_{T}^{(\varepsilon)}\right)\right]
$$

for all $0 \leq \varepsilon \leq 1$, where $\xi^{(\varepsilon)}=\varepsilon \xi^{\prime}+(1-\varepsilon) \xi^{*}$ (here, $\xi^{\prime}$ is a fixed element in $\bar{\Gamma}$ ). The last inequality follows from the fact that, by Proposition 5.1 (c), $\xi^{*}$ can be approximated by elements $\left\{\xi^{(n)}\right\}_{n \geq 1}$ in $\bar{\Gamma}$ in the sense that $\xi_{T}^{(n)} \rightarrow \xi_{T}^{*}$ a.s. Thus, $\xi^{(\varepsilon)}$ can be approximated by the elements $\xi^{(\varepsilon, n)}:=$ $\varepsilon \xi^{\prime}+(1-\varepsilon) \xi^{(n)}$ in $\bar{\Gamma}$, for which we know that

$$
\mathbb{E}\left[\tilde{U}\left(y \xi_{T}^{*}\right)\right] \leq \mathbb{E}\left[\tilde{U}\left(y \xi_{T}^{(\varepsilon, n)}\right)\right]
$$

Passing to the limit as $n \rightarrow \infty$, we obtain (6.4).

In particular we conclude that condition (6.2) is sufficient for both the existence of the solution to the primal problem and its characterization in terms of the dual solution $\xi^{*} \in \widetilde{\Gamma}$ of the dual problem induced by $\Gamma=\bar{\Gamma}$. We now further know that $\xi^{*}$ belongs to the class $\widetilde{\Gamma} \cap \Gamma(\mathcal{S})$ defined in (4.3), and hence, enjoys an explicit parametrization of the form

$$
\xi^{*}:=\varepsilon\left(\int_{0} G^{*}(s) d W_{s}+\int_{0} \int_{\mathbb{R}_{0}} F^{*}(s, z) \widetilde{N}(d s, d z)-\int_{0} a_{s}^{*} d s\right)
$$

for some triple $\left(G^{*}, F^{*}, a^{*}\right)$. 


\section{(ii) Market driven by general additive models}

Our analysis can be extended to more general multidimensional models driven by additive processes (i.e., processes with independent, possibly nonstationary increments; cf. Sato [15] and Kallenberg [20]). For instance, let $(\Omega, F, \mathbb{P})$ be a complete probability space on which is defined a ddimensional additive process $Z$ with Lévy-Itô decomposition:

$$
Z_{t}=\alpha t+\Sigma W_{t}+\int_{0}^{t} \int_{\{\|z\|>1\}} z N(d s, d z)+\int_{0}^{t} \int_{\{\|z\| \leq 1\}} z \widetilde{N}(d s, d z)
$$

where $W$ is a standard $d$-dimensional Brownian motion, $N(d t, d z)$ is an independent Poisson random measure on $\mathbb{R}_{+} \times \mathbb{R}^{d}$, and $\widetilde{N}(d t, d z)=N(d t, d z)-\mathbb{E} N(d t, d z)$. Consider a market model consisting of $n+1$ securities: one risk free bond with price

$$
d B_{t}:=r_{t} B_{t} d t, \quad B_{0}=1, \quad t \geq 0,
$$

and $n$ risky assets with prices determined by the following stochastic differential equations with jumps:

$$
d S_{t}^{i}=S_{t^{-}}^{i}\left\{b_{t}^{i} d t+\sum_{j=1}^{d} \sigma_{t}^{i j} d W_{t}^{j}+\int_{\mathbb{R}^{d}} v^{i}(t, z) \widetilde{N}(d s, d z)\right\}, \quad i=1, \ldots, n,
$$

where the processes $r, b, \sigma$, and $v$ are predictable satisfying usual integrability conditions (cf. Kunita [10]). We assume that $\mathcal{F}:=\mathcal{F}_{\infty^{-}}$, where $\mathbb{F}:=\left\{\mathcal{F}_{t}\right\}_{t \geq 0}$ is the natural filtration generated by $W$ and $N$; namely, $\mathcal{F}_{t}:=\sigma\left(W_{s}, N([0, s] \times A): s \leq t, A \in \mathbb{B}\left(\mathbb{R}^{d}\right)\right)$. The crucial property, particular to this market model, that makes our analysis valid, is the representation theorem for local martingales relative to $Z$ (see [17, Theorem III.4.34]). The definition of the dual class $\Gamma$ given in Section 4 will remain unchanged, and only very minor details will change in the proof of Theorem A.3. Some of the properties of the results in Section 4 regarding the properties of $\Gamma$ will also change slightly. We remark that, by taking a real (nonhomogeneous) Poisson process, the model and results of Chapter 3 in $\mathrm{Xu}$ [5] will be greatly extended. We do not pursue the details here due to the limitation of the length of this paper.

\section{(iii) Optimal wealth-consumption problem}

Another classical portfolio optimization in the literature is that of optimal wealthconsumption strategies under a budget constraint. Namely, we allow the agent to spend money outside the market, while maintaining "solvency" throughout $[0, T]$. In that case the agent aims to maximize the cost functional that contains a "running cost":

$$
\mathbb{E}\left[U_{1}\left(V_{T}\right)+\int_{0}^{T} U_{2}\left(t, c_{t}\right) d t\right]
$$


where $c$ is the instantaneous rate of consumption. To be more precise, the cumulative consumption at time $t$ is given by $C_{t}:=\int_{0}^{t} c_{u} d u$ and the (discounted) wealth at time $t$ is given by

$$
V_{t}=w+\int_{0}^{t} \beta_{u} d S_{u}-\int_{0}^{t} c_{u} d u
$$

Here, $U_{1}$ is a (state-dependent) utility function and $U_{2}(t, \cdot)$ is a utility function for each $t$. The dual problem can now be defined as follows:

$$
v_{\Gamma}(y)=\inf _{\xi \in \Gamma} \mathbb{E}\left[\tilde{U}_{1}\left(y \xi_{T}\right)+\int_{0}^{T} \tilde{U}_{2}\left(s, y \xi_{s}\right) d s\right],
$$

over a suitable class of supermartingales $\Gamma$. For instance, if the support of $v$ is $[-1, \infty)$, then $\Gamma$ can be all supermartingales $\xi$ such that $0 \leq \xi_{0} \leq 1$ and $\left\{\xi_{t} S_{t}\right\}_{t \leq T}$ is a supermartingale. The dual Theorem 3.5 can be extended for this problem. However, the existence of a wealthconsumption strategy pair $(\beta, c)$ that attains the potential final wealth induced by the optimal dual solution (as in Section 5) requires further work. We hope to address this problem in a future publication.

\section{Appendices}

\section{A. Convex Classes of Exponential Supermartingales}

The goal of this part is to establish the theoretical foundations behind Theorem 4.1. We begin by recalling an important optional decomposition theorem due to Föllmer and Kramkov [12]. Given a family of supermartingales $\mathcal{S}$ satisfying suitable conditions, the result characterizes the nonnegative exponential local supermartingales $\xi:=\xi_{0} \varepsilon(X-A)$, where $X \in \mathcal{S}$ and $A \in$ $\mathcal{V}^{+}$, in terms of the so-called upper variation process for $\mathcal{S}$. Concretely, let $D(\mathcal{S})$ be the class of probability measures $\mathbb{Q} \sim \mathbb{P}$ for which there is an increasing predictable process $\left\{A_{t}\right\}_{t \geq 0}$ (depending on $\mathbb{Q}$ and $\mathcal{S}$ ) such that $\left\{X_{t}-A_{t}\right\}_{t \geq 0}$ is a local supermartingale under $\mathbb{Q}$, for all $X \in \mathcal{S}$. The smallest of such processes $A$ is denoted by $A^{\mathcal{S}}(\mathbb{Q})$ and is called the upper variation process for $\mathcal{S}$ corresponding to $\mathbb{Q}$. For easy reference, we state Föllmer and Kramkov's result (see [12] for a proof).

Theorem A.1. Let $\mathcal{S}$ be a family of semimartingales that are null at zero, and that are locally bounded from below. Assume that $0 \in \mathcal{S}$, and that the following conditions hold:

(i) $\mathcal{S}$ is predictably convex,

(ii) $S$ is closed under the Émery distance,

(iii) $p(\mathcal{S}) \neq \emptyset$,

then, the following two statements are equivalent for a nonnegative process $\xi$ :

(1) $\xi$ is of the form $\xi=\xi_{0} \varepsilon(X-A)$, for some $X \in \mathcal{S}$ and an increasing process $A \in \mathcal{U}^{+}$;

(2) $\xi / \mathcal{E}\left(A^{\mathcal{S}}(\mathbb{Q})\right)$ is a supermartingale under $\mathbb{Q}$ for each $\mathbb{Q} \in D(\mathcal{S})$. 
The next result is a direct consequence of the previous representation. Recall that a sequence of processes $\left\{\xi^{n}\right\}_{n \geq 1}$ is said to be "Fatou convergent on $\pi^{\prime \prime}$ to a process $\xi$ if $\left\{\xi^{n}\right\}_{n \geq 1}$ is uniformly bounded from below and it holds that

$$
\xi_{t}=\limsup _{s \downarrow t: s \in \pi} \limsup _{n \rightarrow \infty} \xi_{s}^{n}=\lim _{s \downarrow t: s \in \pi} \inf _{n \rightarrow \infty} \operatorname{limf}_{n \rightarrow \infty} \xi_{s}^{n}
$$

almost surely for all $t \geq 0$.

Proposition A.2. If $\mathcal{S}$ is a class of semimartingales satisfying the conditions in Theorem A.1, then

$$
\Gamma(\mathcal{S}):=\left\{\xi:=\xi_{0} \mathcal{\varepsilon}(X-A): X \in \mathcal{S}, A \text { increasing, and } \xi \geq 0\right\}
$$

is convex and closed under Fatou convergence on any fixed dense countable set $\pi$ of $\mathbb{R}_{+}$; that is, if $\left\{\xi^{n}\right\}_{n \geq 1}$ is a sequence in $\Gamma(\mathcal{S})$ that is Fatou convergent on $\pi$ to a process $\xi$, then $\xi \in \Gamma(\mathcal{S})$.

Proof. The convexity of $\Gamma(\mathcal{S})$ is a direct consequence of Theorem A.1, since the convex combination of supermartingales remains a supermartingale. Let us prove the closure property. Fix a $\mathbb{Q} \in \mathcal{D}(\mathcal{S})$ and denote $C_{t}:=\mathcal{E}\left(A^{\mathcal{S}}(\mathbb{Q})\right)$. Notice that $C_{t}>0$ because $A^{\mathcal{S}}(\mathbb{Q})_{t}$ is increasing and hence, its jumps are nonnegative. Since $\xi^{n} \in \Gamma(\mathcal{S}),\left\{C_{t}^{-1} \xi_{t}^{n}\right\}_{t \geq 0}$ is a supermartingale under $\mathbb{Q}$. Then, for $0<s^{\prime}<t^{\prime}$,

$$
\mathbb{E}^{\mathbb{Q}}\left[C_{t^{\prime}}^{-1} \xi_{t^{\prime}}^{n} \mid \mathscr{F}_{s^{\prime}}\right] \leq C_{s^{\prime}}^{-1} \xi_{s^{\prime}}^{n}
$$

By Fatou's lemma and the right-continuity of process $C$,

$$
\mathbb{E}^{\mathbb{Q}}\left[C_{t}^{-1} \xi_{t} \mid \mathscr{F}_{s^{\prime}}\right]=\mathbb{E}^{\mathbb{Q}}\left[\liminf _{t^{\prime} \downarrow t: t^{\prime} \in \pi} \liminf _{n \rightarrow \infty} C_{t^{\prime}}^{-1} \xi_{t^{\prime}}^{n} \mid \mathcal{F}_{s^{\prime}}\right] \leq C_{s^{\prime}}^{-1} \xi_{s^{\prime}}^{n}
$$

Finally, using the right continuity of the filtration, we have

$$
\mathbb{E}^{\mathbb{Q}}\left[C_{t}^{-1} \xi_{t} \mid \Psi_{s}\right] \leq \lim _{s^{\prime} \backslash s: s^{\prime} \in \pi} \liminf _{n \rightarrow \infty} C_{s^{\prime}}^{-1} \xi_{s^{\prime}}^{n}=C_{s}^{-1} \xi_{s}
$$

where $0 \leq s<t$. Since $\mathbb{Q}$ is arbitrary, the characterization of Theorem A.1 implies that $\xi \in$ $\Gamma(\mathcal{S})$.

The most technical condition in Theorem A.1 is the closure property under Émery distance. The following result is useful to deal with this condition. It shows that the class of integrals with respect to a Poisson random measure is closed with respect to Émery distance, thus extending the analog property for integrals with respect to a fixed semimartingale due to Mémin [13]. 
Theorem A.3. Let $\Theta$ be a closed convex subset of $\mathbb{R}^{2}$ containing the origin. Let $\Pi$ be the set of all predictable processes $(F, G), F \in G_{\mathrm{loc}}(N)$, and $G \in L_{\mathrm{loc}}^{2}(W)$, such that $F(t, \cdot)=G(t)=0$, for all $t \geq T$, and $(F(\omega, t, z), G(\omega, t)) \in \Theta$, for $\mathbb{P} \times d t \times v(d z)$-a.e. $(\omega, t, z) \in \Omega \times \mathbb{R}_{+} \times \mathbb{R}_{0}$. Then, the class

$$
\mathcal{S}:=\left\{X_{t}:=\int_{0}^{t} G(s) d W_{s}+\int_{0}^{t} \int_{\mathbb{R}_{0}} F(s, z) \widetilde{N}(d s, d z):(F, G) \in \Pi\right\}
$$

is closed under convergence with respect to Emery's topology.

Proof. Consider a sequence of semimartingales

$$
X^{n}(t):=\int_{0}^{t} G^{n}(s) d W_{s}+\int_{0}^{t} \int_{\mathbb{R}} F^{n}(s, z) \widetilde{N}(d s, d z), \quad n \geq 1,
$$

in the class $\mathcal{S}$. Let $X$ be a semimartingale such that $X^{n} \rightarrow X$ under Émery topology. To prove the result, we will borrow some results in [13].

For some $\mathbb{Q} \sim \mathbb{P}$, we denote $\mathcal{M}^{2}(\mathbb{Q})$ to be the Banach space of all $\mathbb{Q}$-square integrable martingales on $[0, T]$, endowed with the norm $\|M\|_{\mathcal{M}^{2}(\mathbb{Q})}:=\left(\mathbb{E}^{\mathbb{Q}}\langle M, M\rangle_{T}\right)^{1 / 2}=$ $\left(\mathbb{E}^{\mathbb{Q}}[M, M]_{T}\right)^{1 / 2}$, and $\mathscr{A}(\mathbb{Q})$ to be the Banach space of all predictable processes on $[0, T]$ that have $\mathbb{Q}$-integrable total variations, endowed with the norm $\|A\|_{\mathscr{A}(\mathbb{Q})}:=\mathbb{E}^{\mathbb{Q}} \operatorname{Var}(A)$. Below, $\mathcal{A}_{\text {loc }}^{+}(\mathbb{Q})$ stands for the localized class of increasing process in $\mathcal{A}(\mathbb{Q})$. By [13, Theorem II.3], one can extract a subsequence from $\left\{X^{n}\right\}$, still denote it by $\left\{X^{n}\right\}$, for which one can construct a probability measure $\mathbb{Q}$, defined on $\mathscr{f}_{T}$ and equivalent to $\mathbb{P}_{T}$ (the restriction of $\mathbb{P}$ on $\mathscr{f}_{T}$ ), such that the following assertions hold:

(i) $\xi:=d \mathbb{Q} /\left(d \mathbb{P}_{T}\right)$ is bounded by a constant;

(ii) $X_{t}^{n}=M_{t}^{n}+A_{t}^{n}, t \leq T$, for Cauchy sequences $\left\{M^{n}\right\}_{n \geq 1}$ and $\left\{A^{n}\right\}_{n \geq 1}$ in $\mathcal{M}^{2}(\mathbb{Q})$ and $\mathscr{A}(\mathbb{Q})$, respectively.

Let us extend $M^{n}$ and $A^{n}$ to $[0, \infty)$ by setting $M_{t}^{n}=M_{t \wedge T}^{n}$ and $A^{n}=A_{t \wedge T}^{n}$ for all $t \geq 0$. Also, we extend $\mathbb{Q}$ for $A \in \mathcal{F}$ by setting $\mathbb{Q}(A):=\int_{A} \xi d \mathbb{P}$, so that $\mathbb{Q} \sim \mathbb{P}($ on $\mathcal{F}$ ). In that case, it can be proved that $\mathcal{A}_{\text {loc }}^{+}(\mathbb{P})=\mathcal{A}_{\text {loc }}^{+}(\mathbb{Q})$. This follows essentially from [17, Proposition III.3.5] and Doob's Theorem. Now, let $\xi_{t}:=\left(\left.d \mathbb{Q}\right|_{f_{t}}\right) /\left(\left.d \mathbb{P}\right|_{\mathcal{F}_{t}}\right)=\mathbb{E}\left[\xi \mid \mathcal{F}_{t}\right]$, denote the density process. Since $\xi$ is bounded, both $\left\{\xi_{t}\right\}_{t}$ and $\left\{\left|\Delta \xi_{t}\right|\right\}_{t}$ are bounded. By [17, Lemma III.3.14 and Theorem III.3.11], the $\mathbb{P}$-quadratic covariation $\left[X^{n}, \xi\right]$ has $\mathbb{P}$-locally integrable variation and the unique canonical decomposition $M^{n}+A^{n}$ of $X^{n}$ relative to $\mathbb{Q}$ is given by

$$
M^{n}=X^{n}-\int_{0}^{t} \frac{1}{\xi_{s-}} d\left\langle X^{n}, \xi\right\rangle_{s^{\prime}} \quad A^{n}=\int_{0}^{t} \frac{1}{\xi_{s-}} d\left\langle X^{n}, \xi\right\rangle_{s} .
$$

Also, the $\mathbb{P}$-quadratic variation of the continuous part $X^{n, c}$ of $X^{n}$ (relative to $\mathbb{P}$ ), given by $\left\langle X^{n, c}, X^{n, c}\right\rangle .=\int_{0}\left(G^{n}(s)\right)^{2} d s$, is also a version of the $\mathbb{Q}$-quadratic variation of the continuous part of $X^{n}$ (relative to $\mathbb{Q}$ ). By the representation theorem for local martingales relative to $Z$ (see, e.g., [17, Theorem III.4.34] or [11, Theorem 2.1]), $\xi$ has the representation

$$
\xi_{t}=1+\int_{0}^{t} \xi_{s^{-}} E(s) d W_{s}+\int_{0}^{t} \int_{\mathbb{R}} \xi_{s^{-}} D(s, z) \widetilde{N}(d s, d z)
$$


for predictable $D$ and $E$ necessarily satisfying that $D>-1$,

$$
\mathbb{E} \int_{0}^{T} \int_{\mathbb{R}} D^{2}(s, z) \xi_{s}^{2} v(d z) d s<\infty, \quad \mathbb{E} \int_{0}^{T} E^{2}(s) \xi_{s}^{2} d s<\infty
$$

Then,

$$
\begin{aligned}
\left\langle X^{n}, \xi\right\rangle_{t} & =\int_{0}^{t} G^{n}(s) E(s) \xi_{s^{-}} d s+\int_{0}^{t} \int_{\mathbb{R}} F^{n}(s, z) D(s, z) \xi_{s^{-}} v(d z) d s \\
A_{t}^{n} & =\int_{0}^{t} \int_{\mathbb{R}} F^{n}(s, z) D(s, z) v(d z) d s+\int_{0}^{t} G^{n}(s) E(s) d s
\end{aligned}
$$

We conclude that $\Delta M_{t}^{n}=\Delta X_{t}^{n}=F^{n}\left(t, \Delta Z_{t}\right)$. Hence, $\Delta M^{n}=\Delta \widetilde{M}^{n}$, where $\widetilde{M}^{n}$ is the purely discontinuous local martingale (relative to $\mathbb{Q}$ ) defined by

$$
\widetilde{M}_{t}^{n}:=\int_{0}^{t} \int_{\mathbb{R}} F^{n}(s, z)\left(N(d s, d z)-v^{\mathbb{Q}}(d s, d z)\right),
$$

where $\mathcal{v}^{\mathbb{Q}}(d s, d z):=\Upsilon(s, z) d s v(d z)$ is the compensator of $N$ relative to $\mathbb{Q}$ (see [17, Theorem III.3.17]). It can be shown that $Y=1+D$. Notice that $\widetilde{M}^{n}$ is well defined since $\mathcal{A}_{\mathrm{loc}}^{+}(\mathbb{P})=\mathcal{A}_{\mathrm{loc}}^{+}(\mathbb{Q})$ and [17, Definition III.1.27]. Then, the purely discontinuous part of the local martingale $M^{n}$ (relative to $\mathbb{Q}$ ) is given by $\widetilde{M}^{n}$ (see [17, Corollary I.4.19]), and since $M^{n} \in \mathcal{M}^{2}(\mathbb{Q})$,

$$
\mathbb{E}^{\mathbb{Q}}\left[M^{n}, M^{n}\right]_{T}=\mathbb{E}^{\mathbb{Q}} \int_{0}^{T}\left(F^{n}(s, z)\right)^{2} Y(s, z) \mathcal{v}(d z) d s+\mathbb{E}^{\mathbb{Q}} \int_{0}^{T}\left(G^{n}(s)\right)^{2} d s<\infty .
$$

Similarly, since $\left\{M^{n}\right\}_{n \geq 1}$ is a Cauchy sequences under the norm $\mathbb{E}^{\mathbb{Q}}[M, M]_{T}$,

$$
\begin{aligned}
\mathbb{E}^{\mathbb{Q}}\left[M^{n}-M^{m}, M^{n}-M^{m}\right]_{T}= & \mathbb{E}^{\mathbb{Q}} \int_{0}^{T}\left(F^{n}(s, z)-F^{m}(s, z)\right)^{2} Y(s, z) v(d z) d s \\
& +\mathbb{E}^{\mathbb{Q}} \int_{0}^{T}\left(G^{n}(s)-G^{m}(s)\right)^{2} d s \longrightarrow 0,
\end{aligned}
$$

as $n, m \rightarrow \infty$. Using the notation $\widetilde{\Omega}:=\Omega \times \mathbb{R}_{+} \times \mathbb{R}$ and $\tilde{p}:=p \times \mathbb{B}(\mathbb{R})$, where $p$ is the predictable $\sigma$-field, we conclude that $\left\{F^{n}\right\}_{n \geq 1}$ is a Cauchy sequence in the Banach space

$$
\mathbb{H}_{d}:=\mathbb{L}^{2}(\widetilde{\Omega}, \tilde{p}, Y d \mathbb{Q} d v d t) \cap \mathbb{L}^{1}(\widetilde{\Omega}, \tilde{p},|D| d \mathbb{Q} d v d t),
$$

and thus, there is $F \in \mathbb{H}_{d}$ such that $F^{n} \rightarrow F$, as $n \rightarrow \infty$. Similarly, there exists a $G$ in the Banach space

$$
\mathbb{H}_{c}:=\mathbb{L}^{2}\left(\Omega \times \mathbb{R}_{+}, p, d \mathbb{Q} d t\right) \cap \mathbb{L}^{1}\left(\Omega \times \mathbb{R}_{+}, p,|E| d \mathbb{Q} d v d t\right),
$$


such that $G^{n} \rightarrow G$, as $n \rightarrow \infty$. In particular, $(F, G)$ satisfies condition (iv) since $Y=1+D$ is strictly positive, and each $\left(F^{n}, G^{n}\right)$ satisfies (iv). Also, $F \in G_{\text {loc }}(N)$ relative to $\mathbb{Q}$ in light of $\mathcal{A}_{\mathrm{loc}}^{+}(\mathbb{P})=\mathcal{A}_{\mathrm{loc}}^{+}(\mathbb{Q})$. Similarly, $\int_{0} G^{2}(s) d s$ belongs to $\mathcal{A}_{\mathrm{loc}}^{+}(\mathbb{Q})$, and hence, belongs to $\mathcal{A}_{\mathrm{loc}}^{+}(\mathbb{P})$. It follows that the process

$$
\tilde{X}:=\int_{0}^{t} G(s) d W_{s}+\int_{0}^{t} \int_{\mathbb{R}} F(s, z) \widetilde{N}(d s, d z), \quad n \geq 1,
$$

is a well-defined local martingale relative to $\mathbb{P}$. Applying Girsanov's Theorem to $\tilde{X}$ relative to $\mathbb{Q}$ and following the same argument as above, the purely discontinuous local martingale and bounded variation parts of $\tilde{X}$ are, respectively,

$$
\begin{aligned}
M_{t}^{d} & =\int_{0}^{t} \int_{\mathbb{R}} F(s, z)\left(N(d s, d z)-v^{\mathbb{Q}}(d s, d z)\right), \\
A_{t} & =\int_{0}^{t} \int_{\mathbb{R}} F(s, z) D(s, z) v(d z) d s+\int_{0}^{t} G(s) E(s) d s .
\end{aligned}
$$

The continuous part of $\tilde{X}$ has quadratic variation $\int_{0} G^{2}(s) d s$. We conclude that $\tilde{X} \in \mathcal{M}^{2}(\mathbb{Q}) \oplus$ $\mathscr{A}(\mathbb{Q})$ and $X^{n} \rightarrow \tilde{X}$ on $\mathcal{M}^{2}(\mathbb{Q}) \oplus \mathscr{A}(\mathbb{Q})$. Then, $X^{n}$ converges under Émery's topology to $\tilde{X}$ and hence, $X=\tilde{X}$.

\section{B. Proofs of Some Standard Convex Duality Results}

This appendix sketches the proofs of the results in Section 3. The proofs are standard in convex duality and are given only for the sake of completeness.

Proof of Proposition 3.4. For simplicity, we write $v(y)=v_{\Gamma}(y)$. The monotonicity and range of values of $v$ are straightforward. To prove $(2)$, notice that since $\tilde{U}(\cdot ; \omega)$ is convex, nonincreasing, and $\tilde{U}^{\prime}\left(0^{+} ; \omega\right)=-H(\omega)$, we have

$$
\frac{\mathbb{E}[U(H ; \cdot)]-\inf _{\xi} \mathbb{E}[\tilde{U}(y \xi(T))]}{y} \leq \sup _{\xi \in \Gamma} \mathbb{E}[\xi(T) H] .
$$

On the other hand, by the mean value theorem, dominated convergence theorem, (3.3), and the assumptions in Definition 2.3,

$$
\mathbb{E}[H \widehat{\xi}(T)] \leq \liminf _{y \downarrow 0} \frac{\mathbb{E}[U(H ; \cdot)]-v(y)}{y} \leq \sup _{\xi \in \Gamma} \mathbb{E}[\xi(T) H],
$$

for every $\widehat{\xi} \in \Gamma$. Then, (2) is evident. Uniform continuity is straightforward since for any $h$ small enough it holds that

$$
\left|v_{\Gamma}(y+h)-v_{\Gamma}(y)\right| \leq w_{\Gamma}|h|
$$


Part (i) of (4) is well known. Let us turn out to prove (3) and part (ii) in (4). Let $\left\{\xi^{n}\right\}_{n \geq 1} \subset \Gamma \subset \widetilde{\Gamma}$ be such that

$$
\lim _{n \rightarrow \infty} \mathbb{E}\left[\tilde{U}\left(y \xi_{T}^{n}, \omega\right)\right]=v_{\Gamma}(y)
$$

Without loss of generality, one can assume that each process $\xi^{n}$ is constant on $[T, \infty)$. By Lemma 5.2 in [12], there exist $\bar{\xi}^{n} \in \operatorname{conv}\left(\xi^{n}, \xi^{n+1}, \ldots\right), n \geq 1$, and a nonnegative supermartingale $\left\{\tilde{\xi}_{t}\right\}_{t \geq 0}$ with $\tilde{\xi}_{0} \leq 1$ such that $\left\{\bar{\xi}^{n}\right\}_{n \geq 1}$ is Fatou convergent to $\tilde{\xi}$ on the rational numbers $\pi$; namely,

$$
\widetilde{\xi}_{t}=\limsup _{s \downarrow t: s \in \pi} \limsup _{n \rightarrow \infty} \bar{\xi}_{s}^{n}=\liminf _{s \downarrow t: s \in \pi} \liminf _{n \rightarrow \infty} \bar{\xi}_{s}^{n}, \quad \text { a.s. }
$$

for all $t \geq 0$. By Fatou's Lemma, it is not hard to check that $\left\{\tilde{\xi}(t) V_{t}\right\}_{t \leq T}$ is a supermartingale for every admissible portfolio with value process $V$, and hence, $\tilde{\xi} \in \widetilde{\Gamma}$. Next, since the $\xi^{n \prime}$ s are constant on $[T, \infty)$ and $\tilde{U}(\cdot ; \omega)$ is convex, Fatou's Lemma implies that $\mathbb{E}\left[\tilde{U}\left(y \tilde{\xi}_{T}, \omega\right)\right] \leq v_{\Gamma}(y)$ Finally, we need to verify that, when $\Gamma$ is convex, equality above is attained and that $\tilde{\xi}$ can be approximated by elements of $\Gamma$. Both facts are clear since $\left\{\bar{\xi}^{n}\right\} \subset \Gamma$ and $\lim _{n \rightarrow \infty} \bar{\xi}_{T}^{n}=\widetilde{\xi}_{T}$ a.s. Then, by the continuity and boundedness of $\tilde{U}$,

$$
v_{\Gamma}(y) \leq \lim _{n \rightarrow \infty} \mathbb{E}\left[\tilde{U}\left(y \bar{\xi}_{T}^{n} B_{T}^{-1}\right)\right]=\mathbb{E}\left[\tilde{U}\left(y \tilde{\xi}_{T} B_{T}^{-1}, \omega\right)\right] .
$$

Proof of Theorem 3.5. We follow the arguments in [9, Theorem 9.3]. For simplicity let us write $v(y)$ instead of $v_{\Gamma}(y)$. Recall that $w_{\Gamma}:=\sup _{\xi \in \Gamma} \mathbb{E}[\xi(T) H]$ and define $v(0):=\mathbb{E}[U(H ; \omega)]$. In light of Proposition 3.4, the continuous function $f_{z}(y):=v(y)+z y$ satisfies

$$
\lim _{y \downarrow 0} \frac{f_{z}(y)-f_{z}(0)}{y}=-w_{\Gamma}+z<0, \quad f_{z}(\infty)=\infty,
$$

for all $z<w_{\Gamma}$. Thus, $f_{z}(\cdot)$ attains its minimum at some $y(z) \in(0, \infty)$. By Proposition 3.4, we can find a $\xi_{y(z)} \in \tilde{\Gamma}$ such that

$$
v(y(z))=\mathbb{E}\left[\tilde{U}\left(y(z) \xi_{y(z)}(T), \omega\right)\right]
$$

proving the (i) above. Now, consider the function

$$
F(u):=u y(z) z+\mathbb{E}\left[\tilde{U}\left(u y(z) \xi_{y(z)}(T)\right)\right], \quad u>0 .
$$

Since $\xi_{y(z)}$ can be approximated by elements in $\Gamma$, for each $\varepsilon>0$ there exists a $\xi_{y(z)}^{y, \varepsilon} \in \Gamma$ such that

$$
\mathbb{E}\left[\tilde{U}\left(y \xi_{y(z)}(T)\right)\right]>\mathbb{E}\left[\tilde{U}\left(y \xi_{y(z)}^{y, \varepsilon}(T)\right)\right]-\varepsilon
$$


It follows that for each $\varepsilon>0$,

$$
\begin{aligned}
\inf _{u>0} F(u) & \geq \inf _{y>0}\left\{y z+\mathbb{E}\left[\tilde{U}\left(y \xi_{y(z)}^{y, \varepsilon}(T)\right)\right]\right\}-\varepsilon \geq \inf _{y>0}\{y z+v(y)\}-\varepsilon \\
& =y(z) z+\mathbb{E}\left[\tilde{U}\left(y(z) \xi_{y(z)}(T)\right)\right]-\varepsilon .
\end{aligned}
$$

Since $\varepsilon>0$ is arbitrary, the function $F(u)$ attains its minimum at $u=1$. On the other hand, $(F(1+h)-F(1)) / h$ equals

$$
y(z) z+\mathbb{E}\left[\frac{\tilde{U}\left((1+h) y(z) \xi_{y(z)}(T)\right)-\tilde{U}\left(y(z) \xi_{y(z)}(T)\right)}{h}\right]
$$

which converges to

$$
y(z) z-y(z) \mathbb{E}\left[\left(I\left(y(z) \xi_{y(z)}(T)\right) \wedge H\right) \xi_{y(z)}(T)\right]
$$

as $h \rightarrow 0$. Here, we use (3.3) and the dominated convergence theorem. Then,

$$
\mathbb{E}\left[\left(I\left(y(z) \xi_{y(z)}(T)\right) \wedge H\right) \xi_{y(z)}(T)\right]=z .
$$

This proves (ii) of the theorem, and also (iii) in light of (3.4) and (3.6).

\section{Acknowledgments}

The first author is partially supported by the NSF Grant no. DMS 0906919. This author would like to thank the Department of Statistics and Applied Probability at the University of California at Santa Barbara for its hospitality and support while this paper was in preparation. The second author is supported in part by the NSF Grant no. DMS 0806017.

\section{References}

[1] F. Delbaen and W. Schachermayer, "A general version of the fundamental theorem of asset pricing," Mathematische Annalen, vol. 300, no. 1, pp. 463-520, 1994.

[2] D. O. Kramkov, "Optional decomposition of supermartingales and hedging contingent claims in incomplete security markets," Probability Theory and Related Fields, vol. 105, no. 4, pp. 459-479, 1996.

[3] H. Föllmer and P. Leukert, "Efficient hedging: cost versus shortfall risk," Finance and Stochastics, vol. 4, pp. 117-146, 2000.

[4] J. Cvitanić, "Minimizing expected loss of hedging in incomplete and constrained markets," SIAM Journal on Control and Optimization, vol. 38, no. 4, pp. 1050-1066, 2000.

[5] M. Xu, Minimizing shortfall risk using duality approach-an application to partial hedging in incomplete markets, Ph.D. thesis, Carnegie Mellon University, April 2004.

[6] I. Karatzas and G. Žitković, "Optimal consumption from investment and random endowment in incomplete semimartingale markets," Annals of Probability, vol. 31, no. 4, pp. 1821-1858, 2003.

[7] R. C. Merton, "Lifetime portfolio selection under uncertainty: the continuous-time case," The Review of Economics and Statistics, vol. 51, pp. 247-257, 1969.

[8] R. C. Merton, "Optimum consumption and portfolio rules in a continuous-time model," Journal of Economic Theory, vol. 3, no. 4, pp. 373-413, 1971. 
[9] I. Karatzas, J. P. Lehoczky, S. E. Shreve, and G.-L. Xu, "Martingale and duality methods for utility maximization in an incomplete market," SIAM Journal on Control and Optimization, vol. 29, no. 3, pp. 702-730, 1991.

[10] H. Kunita, "Variational equality and portfolio optimization for price processes with jumps," in Stochastic Processes and Applications to Mathematical Finance, pp. 167-194, 2004.

[11] H. Kunita, "Representation of martingales with jumps and applications to mathematical finance," in Stochastic Analysis and Related Topics in Kyoto, Advanced Studies in Pure Mathematics, pp. 209-232, Math. Soc. Japan, Tokyo, Japan, 2004.

[12] H. Föllmer and D. Kramkov, "Optional decompositions under constraints," Probability Theory and Related Fields, vol. 109, no. 1, pp. 1-25, 1997.

[13] J. Mémin, "Espaces de semi martingales et changement de probabilité," Zeitschrift für Wahrscheinlichkeitstheorie und Verwandte Gebiete, vol. 52, no. 1, pp. 9-39, 1980.

[14] D. Kramkov and W. Schachermayer, "The asymptotic elasticity of utility functions and optimal investment in incomplete markets," Annals of Applied Probability, vol. 9, no. 3, pp. 904-950, 1999.

[15] K. Sato, Lévy Processes and Infinitely Divisible Distributions, Cambridge University Press, Cambridge, UK, 1999.

[16] P. Protter, Stochastic Integration and Differentil Equations, Springer, Berlin, Germany, 2nd edition, 2004.

[17] J. Jacod and A. N. Shiryaev, Limit Theorems for Stochastic Processes, Springer, New York, NY, USA, 2003.

[18] E. Hewitt and K. Stromberg, Real and Abstract Analysis, Springer, New York, NY, USA, 1965.

[19] T. R. Hurd, "A note on log-optimal portfolios in exponential Lévy markets," Statistics and Decisions, vol. 22, pp. 225-236, 2004.

[20] O. Kallenberg, Foundations of Modern Probability, Springer, Berlin, Germany, 1997. 


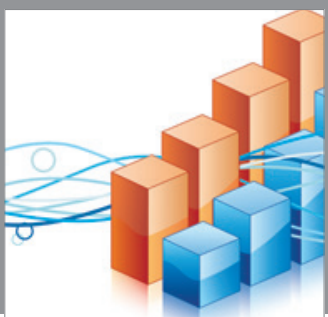

Advances in

Operations Research

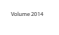

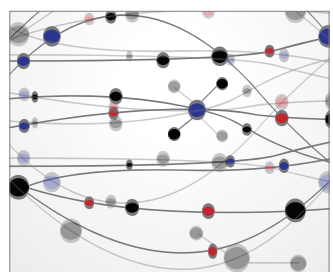

\section{The Scientific} World Journal
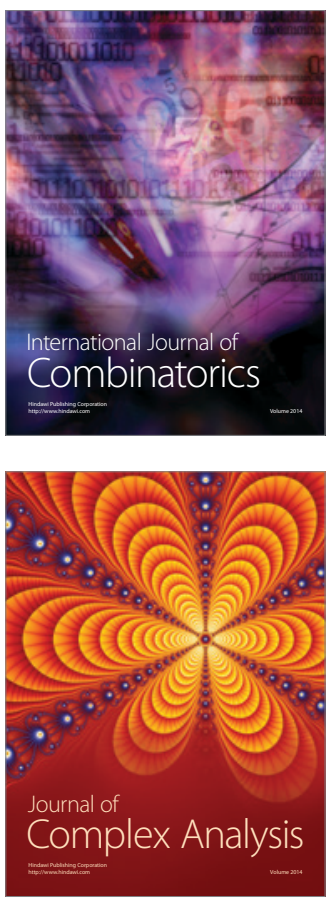

International Journal of

Mathematics and

Mathematical

Sciences
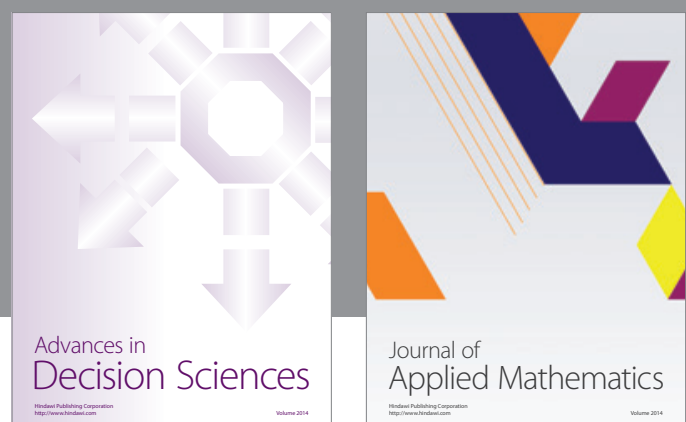

Journal of

Applied Mathematics
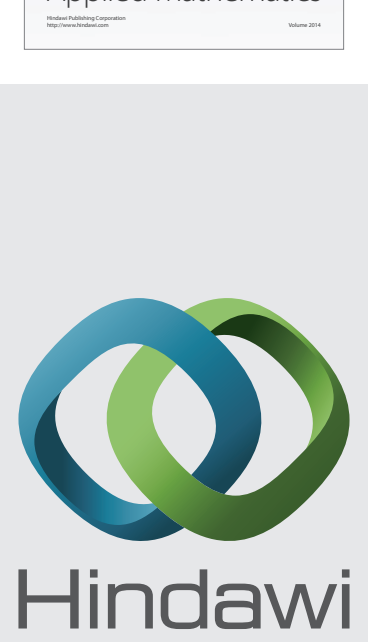

Submit your manuscripts at http://www.hindawi.com
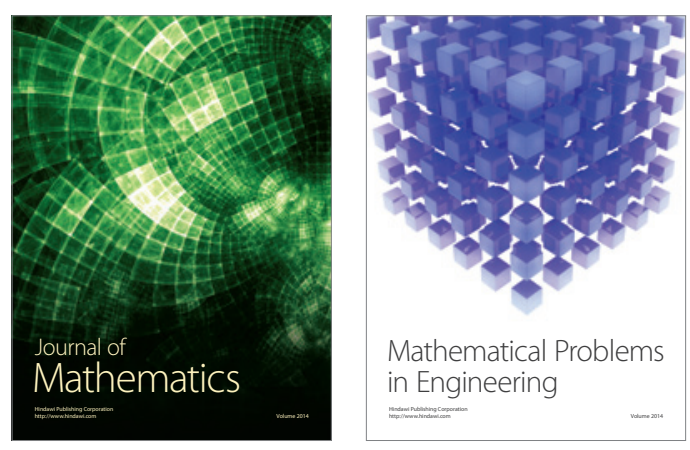

Mathematical Problems in Engineering
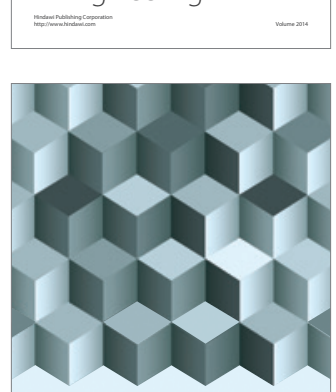

Journal of

Function Spaces
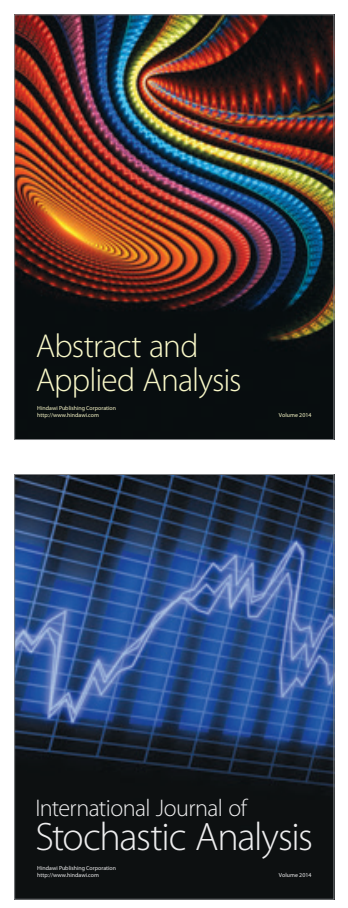

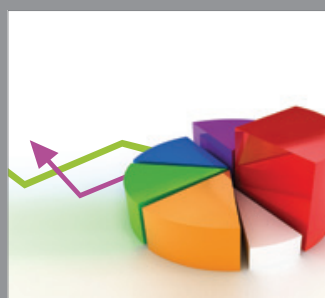

ournal of

Probability and Statistics

Promensencen
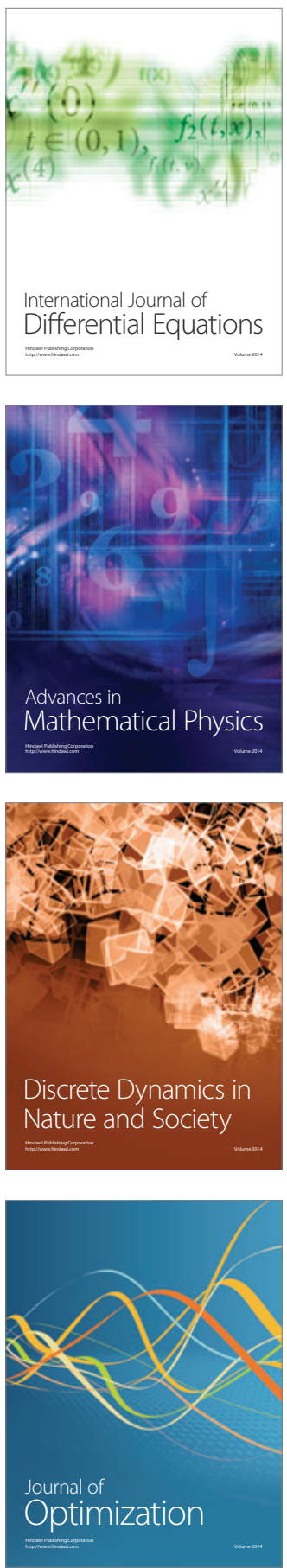